\title{
Petrology of the Creignish Hills Pluton, Cape Breton Island, Nova Scotia
}

\author{
Chris E. White, Sandra M. Barr and R. Mark Campbell \\ Department of Geology, Acadia University, Wolfville, Nova Scotia BOP IXO, Canada
}

Date Received October 6, 1989

Date Accepted March 21, 1990

\begin{abstract}
Mapping and petrologic studies of the Creignish Hills Pluton in the Bras d'Or Terrane of central Cape Breton Island have shown that the pluton consists mainly of tonalite-diorite and coarse-grained monzogranite units, with smaller areas of granodiorite-tonalite, granodiorite-monzogranite, and fine-grained monzogranite. Petrographic and chemical characteristics of the tonalite-diorite suggest that hornblende fractionation ( \pm biotite accumulation) may have produced much of the observed variation in the unit, whereas feldspar fractionation was the dominant process producing variation within each of the other units. The tonalite-diorite unit is interpreted to be late Precambrian (ca. $560 \mathrm{Ma}$ ) in age like most other dioritic and tonalitic plutons in the Bras d'Or Terrane. It is probably not co-genetic with the intermediate and felsic units of the pluton. The latter may be of Early Ordovician age (ca.495 Ma) on the basis of petrological similarities of the coarse-grained monzogranite unit to the Cape Smoky and Kellys Mountain monzogranites. Like other Bras d'Or Terrane plutons, the Creignish Hills Pluton probably formed in a continental margin volcanic arc setting during and after late Precambrian to Early Cambrian subduction.
\end{abstract}

Des travaux de cartographie et de pétrologie ont permis d'établir que le pluton de Creignish Hills (Lanière de Bras d'Or, centre de l'île du Cap Breton) se compose surtout de tonalite-diorite et d'unités de monzogranite à grain grossier, avec des îlots de granodiorite-tonalite, granodiorite-monzogranite et monzogranite à grain fin. Les caractères de sa pétrographie et de son chimisme suggèrent que le variation présente au sein de la tonalite-diorite émane pour une grande part du fractionnement de la homblende ( \pm accumulation de biotite). En revanche, la variation observée dans chacune des autres unités provient surtout du fractionnement du feldspath. On interprète l'âge de l'unité de tonalite-diorite comme étant précambrien tardif (env. $560 \mathrm{Ma}$ ), âge qu'ont en commun la plupart des plutons dioritiques et tonalitiques au sein de la Lanière de Bras d'Or. Cette unité n'est probablement pas cogénétique avec les unités intermédiaires et felsiques du pluton. Ces dernières pourraient remonter au début de l'Ordovicien (env. $495 \mathrm{Ma}$ ) si l'on se fie aux similitudes pétrologiques entre l'unité de monzogranite à grain grossier et les monzogranites de Cape Smoky et de Kellys Mountain. A l'instar des autres plutons de la Lanière de Bras d'Or, le pluton de Creignish Hills s'est probablement formé en contexte d'arc insulaire sur une marge continentale pendant et après la subduction tardiprécambrienne à éocambrienne.

[Traduit par le joumal]

\section{INTRODUCTION}

The Creignish Hills Pluton is a large composite dioritic to granitic intrusion forming much of the southern part of the Creignish Hills in western Cape Breton Island (Fig. 1). This area is part of the Bras d'Or Terrane, a recently proposed tectonostratigraphic division of the northern Appalachian Orogen (Raeside and Barr, 1988; Barr and Raeside, 1989). The purpose of this paper is to describe the field relations, petrology and geochemistry of the Creignish Hills Pluton, and to compare it to other plutons in the Bras d'Or Terrane of Cape Breton Island.

The Creignish Hills Pluton was included in regional geological mapping by Ferguson and Weeks (1950), Kelley (1967) and Milligan (1970), and was the focus of mapping by Campbell (1980). However, the petrology of the pluton has not been previously described.

\section{GEOLOGICAL SETTING}

The Creignish Hills form a prominent topographic feature with an elevation of about $250 \mathrm{~m}$. Metamorphic rocks form most of the northeastern part of the Creignish Hills, and the Creignish Hills Pluton forms most of the southern part (Fig. 1). The metamorphic rocks, including gneiss, marble, quartzite, slate and minor volcanic rocks, were previously assigned to the George River Group (Kelley, 1967; Milligan, 1970). However, the gneissic rocks have recently been shown to be a separate unit in faulted contact with the other metamorphic rocks (Armitage, 1989; Campbell and Raeside, 1990). Contacts between the Creignish Hills Pluton and the metamorphic rocks are not exposed but interpreted to be intrusive on the basis of increase in size and abundance of xenoliths of metamorphic lithologies in the plutonic rocks near the pluton margins. 


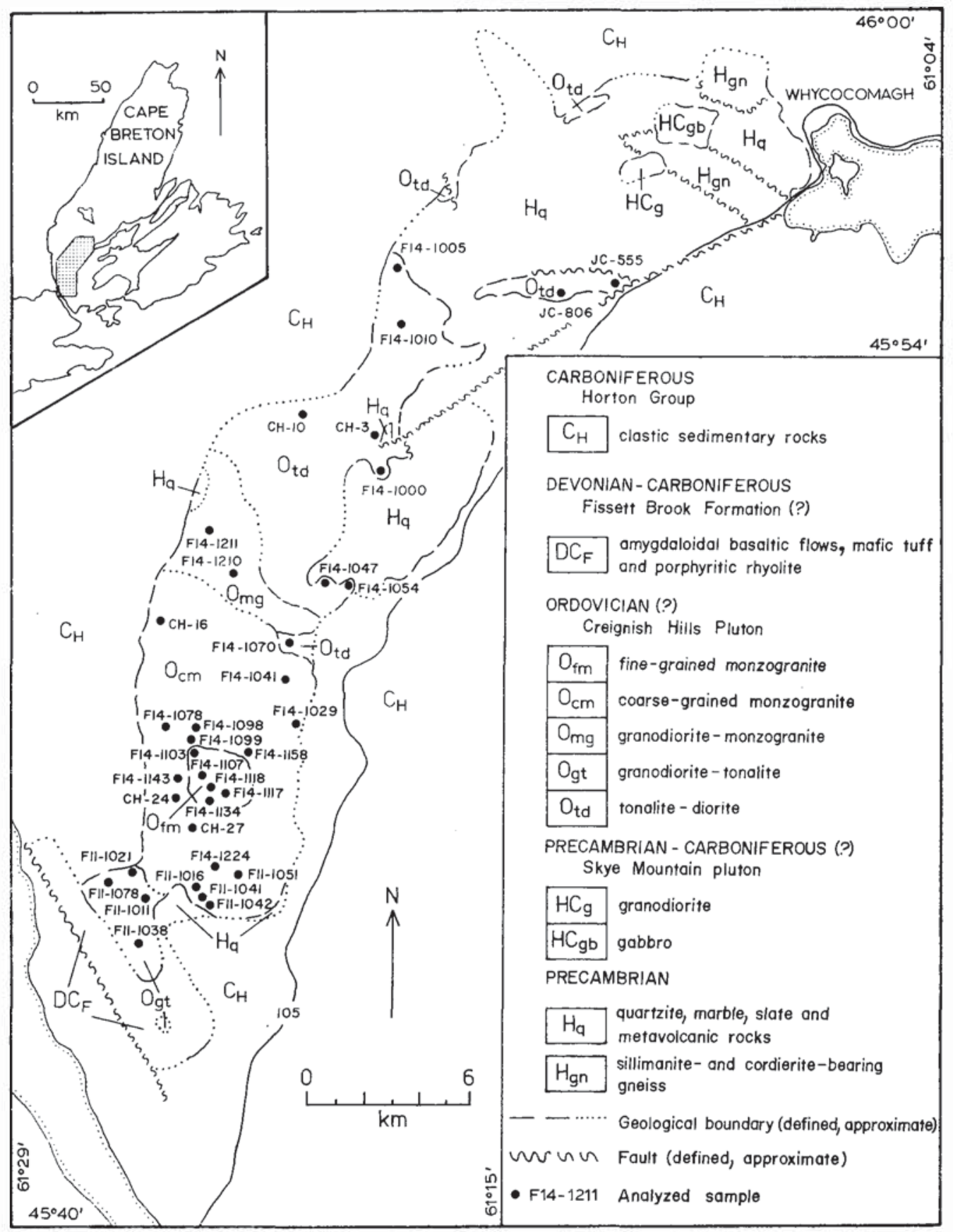

Fig. 1. Geological map of the Creignish Hills (after Campbell, 1980; J.M. Campbell, personal communication, 1989, and the present study).

Volcanic and sedimentary rocks generally assigned to the Devonian-Carboniferous Fissett Brook Formation (Kelley and Mackasey, 1965; Blanchard, 1982) form the southwestern margin of the Creignish Hills Pluton (Fig. 1). Clastic sedimentary rocks of the Lower Carboniferous Horton Group unconformably overlie the other map units around the margins of the Creignish Hills.

\section{PLUTONIC UNITS}

\section{Introduction}

On the basis of this study, the Creignish Hills Pluton is divided into five lithological units: tonalite-diorite, granodiorite- tonalite, granodiorite-monzogranite, coarse-grained monzogranite, and fine-grained monzogranite (Fig. 1). Small tonalitic intrusions within metamorphic rocks to the northeast of the main pluton are interpreted to be part of the tonalite-diorite unit based on lithological similarity. However, intrusions of granodiorite and gabbro (Skye Mountain Pluton) farther east in the Creignish Hills (Fig. 1) are not similar to any lithologies in the Creignish Hills Pluton and hence are not included in the present study.

The Creignish Hills Pluton and adjacent metamorphic rocks are cut by numerous aplite, pegmatite and porphyry dykes that may represent late stages of pluton crystallization. Mafic dykes of uncertain age(s) are also common.

The relative ages of the units of the Creignish Hills Pluton are 
somewhat speculative. The fine-grained monzogranite is considered to be the youngest unit as it has been chilled against and occurs as dykes ( 1 to $2 \mathrm{~m}$ wide) in the coarse-grained monzogranite. The coarse-grained monzogranite appears to have been chilled against the granodiorite-tonalite unit. Nodirect field evidence indicating relative ages of the other units was observed. However, on the basis of $\mathrm{Rb}-\mathrm{Sr}$ and ${ }^{40} \mathrm{Ar} /{ }^{39} \mathrm{Ar}$ data discussed in a subsequent section, the tonalite-diorite is assumed to be the oldest unit.

All of the plutonic units locally exhibit zones of intense fracturing, shearing, and alteration, with the development of secondary chlorite, epidote, and/or hematite.

Average modal compositions of each unit determined by counting a minimum of 200 points in thin sections or slabs stained for K-feldspar (Hutchison, 1973) are shown in Table 1.

\section{Tonalite-diorite}

The tonalite-diorite unit consists mainly of tonalite grada- tional to quartz diorite and diorite, with minor granodiorite and quartz monzodiorite (Fig. 2). The rocks are generally grey in colour and fine- to medium-grained, with hypidiomorphic granular to locally porphyritic textures.

Zoned (An25-50), subhedral plagioclase crystals range in length from $0.5 \mathrm{~mm}$ up to $1 \mathrm{~cm}$, the latter in porphyritic samples. Alteration to sericite and saussurite varies from moderate to intense. Mafic minerals include amphibole, biotite, and rarely pyroxene as relict cores in amphibole. Green pleochroic amphibole is the most abundant mafic mineral and forms anhedral to subhedral grains up to $4 \mathrm{~mm}$ in length that are commonly twinned. Microprobe analyses (Table 2) indicate that the amphibole varies in composition from magnesio-hornblende to edenite (terminology of Leake, 1978). Alteration to actinolite and chlorite has occurred in some samples.

Pleochroic brown biotite is subhedral to anhedral, up to 5 $\mathrm{mm}$ in diameter, and occurs both as separate flakes and in association with amphibole. Most of the biotite grains show evidence of deformation (kinking) and are at least partly altered

Table 1. Average modal compositions (with standard deviations) of major units of the Creignish Hills Pluton.

\begin{tabular}{|c|c|c|c|c|c|c|}
\hline & $\begin{array}{l}\text { Tonalite- } \\
\text { Diorite } \\
(\mathrm{n}=26)\end{array}$ & $\begin{array}{c}\text { Granodiorite- } \\
\text { Tonalite } \\
(\mathrm{n}=17)\end{array}$ & $\begin{array}{c}\text { Granodiorite- } \\
\text { Monzogranite } \\
(\mathrm{n}=18)\end{array}$ & $\begin{array}{l}\text { Coarse-grained } \\
\text { Monzogranite } \\
(\mathrm{n}=34)\end{array}$ & $\begin{array}{l}\text { Fine-grained } \\
\text { Monzogranite } \\
(\mathrm{n}=17)\end{array}$ & $\begin{array}{l}\text { Aplite } \\
\text { Dykes } \\
(\mathrm{n}=7)\end{array}$ \\
\hline Plagioclase & $55.0 \pm 8.4$ & $51.3 \pm 10.5$ & $40.7 \pm 3.9$ & $29.4 \pm 5.9$ & $29.8 \pm 3.8$ & $15.6 \pm 6.1$ \\
\hline K-feldspar & $3.8 \pm 3.3$ & $11.7 \pm 6.0$ & $18.1 \pm 5.3$ & $32.1 \pm 5.2$ & $28.0 \pm 5.1$ & $46.0 \pm 7.5$ \\
\hline Quartz & $17.2 \pm 6.7$ & $28.8 \pm 3.0$ & $32.0 \pm 5.3$ & $33.9 \pm 4.5$ & $37.1 \pm 3.8$ & $36.1 \pm 6.4$ \\
\hline Amphibole & $18.0 \pm 8.4$ & $1.7 \pm 2.1$ & & $0.4 \pm 0.7$ & & \\
\hline Pyroxene & $0.2 \pm 1.1$ & & & & & \\
\hline Biotite & $5.0 \pm 5.7$ & $4.5 \pm 2.9$ & $9.1 \pm 4.2$ & $4.3 \pm 2.8$ & $5.0 \pm 3.1$ & $1.4 \pm 1.2$ \\
\hline $\begin{array}{r}\text { Accessory } \\
\text { minerals }\end{array}$ & $0.7 \pm 1.0$ & $0.8 \pm 1.0$ & $0.2 \pm 0.2$ & & & $0.8 \pm 2.1$ \\
\hline
\end{tabular}



Fig. 2. Ternary plot of modal quartz-plagioclase-potassium feldspar compositions of samples from the Creignish Hills Pluton. Shaded symbols are analyzed samples. Fields from Streckeisen (1976). 
Table 2. Representative amphibole and biotite analyses*.

Tonalite-diorite

(F14-1000)

\begin{tabular}{|c|c|c|c|c|c|c|}
\hline \multirow{2}{*}{$\begin{array}{l}\text { Oxides } \\
\mathrm{SiO}_{2}\end{array}$} & \multicolumn{4}{|c|}{ Amphibole } & \multicolumn{2}{|c|}{ Biotite } \\
\hline & 45.91 & 47.76 & 46.08 & 46.19 & 33.71 & 34.97 \\
\hline $\mathrm{TiO}_{2}$ & 1.04 & 0.82 & 1.22 & 1.09 & 2.72 & 3.71 \\
\hline $\mathrm{Al}_{2} \mathrm{O}_{3}$ & 7.98 & 6.90 & 7.78 & 8.02 & 16.23 & 14.90 \\
\hline $\mathrm{Cr}_{2} \mathrm{O}_{3}$ & 0.00 & 0.00 & 0.00 & 0.00 & 0.00 & 0.00 \\
\hline $\mathrm{FeO}$ & 17.15 & 15.88 & 16.92 & 16.05 & 21.31 & 19.91 \\
\hline $\mathrm{MnO}$ & 0.46 & 0.47 & 0.36 & 0.47 & 0.36 & 0.30 \\
\hline $\mathrm{MgO}$ & 11.94 & 13.26 & 11.62 & 12.14 & 13.73 & 12.67 \\
\hline $\mathrm{CaO}$ & 11.48 & 11.36 & 11.64 & 12.01 & 0.00 & 0.00 \\
\hline $\mathrm{Na}_{2} \mathrm{O}$ & 1.26 & 1.28 & 1.07 & 0.98 & 0.00 & 0.00 \\
\hline $\mathrm{K}_{2} \mathrm{O}$ & 0.88 & 0.74 & 0.95 & 0.82 & 5.11 & 6.68 \\
\hline Total & 98.10 & 98.47 & 97.64 & 97.77 & 93.13 & 93.14 \\
\hline Cations & $0=32$ & $0=32$ & $0=32$ & $0=32$ & $0=24$ & $0=24$ \\
\hline $\mathrm{Si}$ & 6.85 & 7.03 & 6.90 & 6.80 & 5.22 & 5.42 \\
\hline $\mathrm{Al}^{i v}$ & 1.45 & 0.97 & 1.10 & 1.12 & 2.78 & 2.58 \\
\hline Alvi & 0.26 & 0.23 & 0.27 & 0.29 & 0.18 & 0.14 \\
\hline $\mathrm{Ti}$ & 0.12 & 0.09 & 0.14 & 0.12 & 0.32 & 0.43 \\
\hline $\mathrm{Cr}$ & 0.00 & 0.00 & 0.00 & 0.00 & 0.00 & 0.00 \\
\hline $\mathrm{Fe}$ & 2.14 & 1.95 & 2.12 & 2.00 & 2.76 & 2.58 \\
\hline $\mathrm{Mn}$ & 0.06 & 0.06 & 0.05 & 0.06 & 0.05 & 0.04 \\
\hline $\mathrm{Mg}$ & 2.66 & 2.91 & 2.59 & 2.69 & 3.17 & 2.93 \\
\hline $\mathrm{Ca}$ & 1.84 & 1.79 & 1.87 & 1.92 & 0.00 & 0.00 \\
\hline $\mathrm{Na}$ & 0.37 & 0.37 & 0.31 & 0.28 & 0.00 & 0.00 \\
\hline K & 0.17 & 0.14 & 0.18 & 0.16 & 1.01 & 1.32 \\
\hline $\mathrm{Fe} / \mathrm{Fe}+\mathrm{Mg}$ & 0.44 & 0.40 & 0.45 & 0.43 & 0.47 & 0.47 \\
\hline
\end{tabular}

Coarse-grained monzogranite (F14-1158)

\begin{tabular}{|c|c|c|c|c|c|}
\hline \multicolumn{2}{|c|}{ Amphibole } & \multicolumn{2}{|c|}{ Biotite } & \multicolumn{2}{|c|}{ Biotite } \\
\hline 42.03 & 42.81 & 36.38 & 34.86 & 36.21 & 35.87 \\
\hline 0.95 & 1.79 & 4.11 & 3.55 & 4.20 & 3.51 \\
\hline 9.49 & 9.00 & 13.20 & 13.15 & 17.01 & 17.54 \\
\hline 0.00 & 0.17 & 0.05 & 0.00 & 0.00 & 0.00 \\
\hline 28.05 & 26.54 & 29.64 & 29.10 & 20.41 & 21.68 \\
\hline 0.70 & 0.78 & 0.37 & 0.39 & 0.56 & 0.45 \\
\hline 4.44 & 5.48 & 4.78 & 5.81 & 8.34 & 7.60 \\
\hline 10.61 & 10.13 & 0.00 & 0.40 & 0.00 & 0.00 \\
\hline 1.53 & 1.81 & 0.00 & 0.00 & 0.00 & 0.00 \\
\hline 1.36 & 1.31 & 8.94 & 8.76 & 9.27 & 9.68 \\
\hline 99.16 & 99.82 & 97.47 & 96.02 & 96.00 & 96.33 \\
\hline $0=32$ & $0=32$ & $0=24$ & $0=24$ & $0=24$ & $0=24$ \\
\hline 6.59 & 6.61 & 5.71 & 5.62 & 5.51 & 5.48 \\
\hline 1.41 & 1.39 & 2.29 & 2.38 & 2.49 & 2.52 \\
\hline 0.35 & 0.25 & 0.15 & 0.12 & 0.55 & 0.64 \\
\hline 0.11 & 0.21 & 0.49 & 0.43 & 0.48 & 0.40 \\
\hline 0.00 & 0.02 & 0.01 & 0.00 & 0.00 & 0.00 \\
\hline 3.68 & 3.43 & 3.89 & 3.93 & 2.60 & 2.77 \\
\hline 0.09 & 0.10 & 0.05 & 0.05 & 0.07 & 0.06 \\
\hline 1.04 & 1.26 & 1.12 & 1.40 & 1.89 & 1.73 \\
\hline 1.78 & 1.68 & 0.00 & 0.07 & 0.00 & 0.00 \\
\hline 0.47 & 0.54 & 0.00 & 0.00 & 0.00 & 0.00 \\
\hline 0.27 & 0.26 & 1.79 & 1.39 & 1.80 & 1.89 \\
\hline 0.78 & 0.73 & 0.78 & 0.74 & 0.59 & 0.62 \\
\hline
\end{tabular}

*Analyses by electron microprobe (Cambridge Instruments Microscan 5) at Dalhousie University, Halifax, Nova Scotia.

to chlorite and/or epidote. The $\mathrm{Fe} / \mathrm{Fe}+\mathrm{Mg}$ ratio is about 0.47 , similar to that in co-existing amphibole (Table 2).

Minor amounts of interstitial perthitic microcline are present in most samples. Locally it forms phenocrysts up to $5 \mathrm{~mm}$ in diameter and poikilitically encloses plagioclase, biotite, amphibole and quartz. Quartz varies in abundance from $1 \%$ in diorite to more than $20 \%$ in tonalite and granodiorite, and is anhedral and interstitial, with inclusions of plagioclase, amphibole and biotite. It commonly displays undulatory extinction.

Accessory phases include opaque minerals, apatite, titanite and rare zircon. Opaque minerals (magnetite and pyrite) vary in abundance (up to $1 \%$ ) and tend to occur with the mafic minerals.
Granodiorite-tonalite

The granodiorite-tonalite unit forms the southwestem part of the Creignish Hills Pluton and also outcrops in a small area (window?) within the Devonian-Carboniferous unit to the south (Fig. 1). Modal compositions of samples from this unit range from granodiorite to tonalite (Table 1, Fig. 2) with granodiorite more abundant than tonalite.

Plagioclase occurs as subhedral to rarely euhedral laths. Grain length varies from $<1 \mathrm{~mm}$ to $5 \mathrm{~mm}$ with compositions in the range An25-35. Myrmekite occurs locally where plagioclase is in contact with K-feldspar. Anhedral interstitial quartz typi- 
cally displays sutured grain boundaries and undulatory extinction. Perthitic alkali feldspar is also interstitial and in places poikilitic with plagioclase inclusions. Patches of alteration to calcite are common.

Both amphibole and biotite are intensely altered to chlorite and epidote. Accessory phases include abundant opaque minerals, apatite, zircon and rare titanite.

\section{Granodiorite-monzogranite}

The granodiorite-monzogranite unit forms the central part of the pluton between the tonalite-diorite and coarse-grained monzogranite units. Granodiorite apparently grades westward to more monzogranitic compositions. The rocks have fine- to medium-grained, allotrimorphic inequigranular textures, with weak to moderate easterly trending foliation defined by alignment of biotite and feldspar. The origin of the foliation is not known but may have resulted from emplacement of the unit during localized shearing.

Plagioclase is anhedral to subhedral and extensively altered to sericite and minor saussurite. Myrmekite is common. Quartz generally forms discrete grains with undulatory extinction. Microcline occurs as anhedral interstitial grains with inclusions of plagioclase, quartz, and biotite. It ranges in grain length from $<0.25 \mathrm{~mm}$ to over $1 \mathrm{~cm}$ in coarse-grained rocks. Brown pleochroic biotite occurs as small $(<0.25 \mathrm{~mm})$ subhedral flakes moderately to intensely altered to chlorite. No amphibole was observed. Accessory minerals include abundant titanite, opaque minerals, apatite and rare zircon.

\section{Coarse-grained monzogranite}

Coarse-grained monzogranite forms most of the southern part of the Creignish Hills Pluton, an area of more than $50 \mathrm{~km}^{2}$. Modal compositions generally plot in the monzogranite field with a few samples on the margin of the syenogranite field (Fig. 2). Texture is coarse-grained, hypidiomorphic to allotrimorphic granular to slightly porphyritic.

Anhedral to rarely euhedral plagioclase (An20-35) varies in grain length from $<1 \mathrm{~mm}$ to $1 \mathrm{~cm}$ in the slightly porphyritic rocks. Interstitial perthitic microcline is typically the coarsest mineral, with diameters up to $1.5 \mathrm{~cm}$. It is commonly poikilitic with embayed inclusions of plagioclase, quartz and mafic minerals. Quartz forms discrete grains with sutured margins and undulatory extinction. Pleochroic green-brown biotite occurs as individual anhedral to subhedral flakes that are commonly kinked and intensely chloritized. Microprobe analyses show that the biotite is iron-rich (Table 2). Minor pleochroic green, subhedral amphibole occurs in a few samples as glomeroporphyritic clusters partially replaced by chlorite. Microprobe analyses of amphibole (Table 2) indicates a ferro-edenitic hornblende composition (Leake, 1978) with $\mathrm{Fe} / \mathrm{Fe}+\mathrm{Mg}$ ratio similar to that in coexisting biotite.

Accessory phases include apatite, zircon, opaque minerals, and less commonly titanite. Apatite and opaque minerals are associated with homblende and biotite.

\section{Fine-grained monzogranite}

Fine-grained monzogranite forms a sub-elliptical body about $4 \mathrm{~km}^{2}$ in area within the central region of the coarse-grained monzogranite. The modal mineralogy (Table 1) plots exclusively in the monzogranite field (Fig. 2). Texture is fine-grained, allotriomorphic (locally hypidiomorphic) granular, and in places porphyritic. Quartz is more abundant than in the coarse-grained monzogranite. Plagioclase forms anhedral to subhedral laths up to $5 \mathrm{~mm}$ in length in the more porphyritic varieties. It also occurs as interstitial poikilitic grains with inclusions of embayed quartz. Most grains are strongly zoned from about An18 to An40, whereas phenocrysts are more calcic with core compositions about An54. Perthitic microcline forms interstitial anhedral grains with embayed inclusions of quartz and plagioclase.

Biotite occurs in clusters and as separate flakes and is only slightly to moderately chloritized. Microprobe analyses (Table 2) indicate that the biotite has higher $\mathrm{Al}$ and lower $\mathrm{Fe}$ content compared to biotite in the coarse-grained monzogranite. Accessory minerals include apatite, zircon and opaque minerals.

\section{Aplite, pegmatite, and porphyry dykes}

All units of the pluton are cut by numerous small $(<30 \mathrm{~cm}$ to $10 \mathrm{~m}$ wide) aplitic, pegmatitic and porphyritic granite dykes, but they appear to be most abundant in the coarse-grained monzogranite unit. The porphyritic dykes typically contain phenocrysts of quartz, K-feldspar, and/or plagioclase up to $1 \mathrm{~cm}$ in length in a fine-grained groundmass with abundant granophyre and rare biotite. Modal compositions of both aplitic and porphyritic dykes are mainly syenogranite with minor monzogranite and alkali feldspar granite (Fig. 2). Pegmatite dykes are also of syenogranite composition and consist of coarse intergrowths of alkali feldspar and quartz displaying graphic textures with rare biotite flakes.

\section{GEOCHEMISTRY}

Thirty-seven samples from the Creignish Hills Pluton and satellite intrusions were analyzed (Appendix A) for major and trace elements (Tables 3-6). In addition, rare earth element data have been obtained for six samples (Table 7; Appendix A).

The ten analyzed samples from the tonalite-diorite unit range in $\mathrm{SiO}_{2}$ content from 50 to $61 \%$ (Table 3). Major element oxides (e.g., Fig. 3) have negative correlation with $\mathrm{SiO}_{2}$, with the exceptions of $\mathrm{K}_{2} \mathrm{O}$ (positive correlation) and $\mathrm{Na}_{2} \mathrm{O}$ (no correlation). Among the trace elements (e.g., Fig. 4), Ba, Rb, and $\mathrm{Zr}$ show weak positive correlation with $\mathrm{SiO}_{2} ; \mathrm{Sr}, \mathrm{Y}, \mathrm{Zn}, \mathrm{V}, \mathrm{Ga}$, and F show negative correlation, whereas the other elements display little or no correlation with $\mathrm{SiO}_{2}$. Cu shows a wide variation from about 5 to $80 \mathrm{ppm} ; \mathrm{Sn}$ and $U$ values are low.

Samples from the granodiorite-tonalite unit (Table 4) show a narrow range in $\mathrm{SiO}_{2}$ content from 67 to about $70 \%$, little variation in most other major element oxides, and low $\mathrm{K}_{2} \mathrm{O}$ and high $\mathrm{Na}_{2} \mathrm{O}$ contents. Among the trace elements, $\mathrm{Ba}$ and $\mathrm{Sr}$ show considerable spread, as do $\mathrm{Pb}$ and $\mathrm{Zn}$. 
Table 3. Chemical analyses and CIPW normative mineralogy of samples from the tonalite-diorite unit.

\begin{tabular}{|c|c|c|c|c|c|c|c|c|c|c|}
\hline SAMPLE & $\begin{array}{l}\text { F14- } \\
1000\end{array}$ & $\begin{array}{l}\text { F14- } \\
1005\end{array}$ & $\begin{array}{l}\text { F14- } \\
1010\end{array}$ & $\begin{array}{l}\text { F14- } \\
1047\end{array}$ & $\begin{array}{l}\text { F14- } \\
1054\end{array}$ & $\begin{array}{l}\text { F14- } \\
1070\end{array}$ & $\begin{array}{c}\mathrm{CH}-87 \\
003\end{array}$ & $\begin{array}{c}\mathrm{CH}-87 \\
010\end{array}$ & $\begin{array}{c}\text { JC-87 } \\
555\end{array}$ & $\begin{array}{c}\text { JC-87 } \\
806\end{array}$ \\
\hline \multicolumn{11}{|c|}{ MAJOR OXIDES (wt. \%) } \\
\hline $\mathrm{SiO}_{2}$ & 61.20 & 57.90 & 59.90 & 57.10 & 58.40 & 52.40 & 50.06 & 59.30 & 56.31 & 59.25 \\
\hline $\mathrm{TiO}_{2}$ & 0.58 & 0.64 & 0.61 & 0.58 & 0.70 & 0.71 & 0.91 & 0.74 & 0.66 & 0.61 \\
\hline $\mathrm{Al}_{2} \mathrm{O}_{3}$ & 14.5 & 17.5 & 16.8 & 17.8 & 17.5 & 18.8 & 17.88 & 16.99 & 15.30 & 16.77 \\
\hline $\mathrm{Fe}_{2} \mathrm{O}_{3}$ & 2.10 & 3.04 & 2.79 & 2.87 & 2.94 & 5.27 & 1.37 & 0.99 & 1.86 & 1.39 \\
\hline $\mathrm{FeO}$ & 4.05 & 4.10 & 3.70 & 3.90 & 4.10 & 1.65 & 8.37 & 6.10 & 6.11 & 4.58 \\
\hline $\mathrm{MnO}$ & 0.12 & 0.14 & 0.13 & 0.17 & 0.16 & 0.17 & 0.20 & 0.13 & 0.16 & 0.12 \\
\hline $\mathrm{MgO}$ & 4.60 & 3.80 & 3.30 & 3.80 & 3.80 & 4.60 & 5.51 & 3.77 & 5.59 & 3.73 \\
\hline $\mathrm{CaO}$ & 5.60 & 5.80 & 5.70 & 6.20 & 6.20 & 8.20 & 6.90 & 3.97 & 5.49 & 4.96 \\
\hline $\mathrm{Na}_{2} \mathrm{O}$ & 3.1 & 3.8 & 3.5 & 3.3 & 3.5 & 4.6 & 2.94 & 2.60 & 2.28 & 3.25 \\
\hline $\mathrm{K}_{2} \mathrm{O}$ & 3.1 & 2.0 & 2.0 & 1.9 & 2.1 & 1.6 & 1.46 & 1.66 & 2.24 & 2.29 \\
\hline $\mathrm{P}_{2} \mathrm{O}_{5}$ & 0.17 & 0.18 & 0.16 & 0.17 & 0.19 & 0.25 & 0.18 & 0.15 & 0.14 & 0.14 \\
\hline LOI & 1.7 & 1.9 & 1.6 & 2.4 & 1.6 & 2.3 & 2.5 & 2.9 & 2.5 & 2.3 \\
\hline TOTAL OXIDES & 100.82 & 100.80 & 100.19 & 100.19 & 101.19 & 100.55 & 98.28 & 99.30 & 98.64 & 99.39 \\
\hline \multicolumn{11}{|c|}{ NORMATIVE MINERALOGY (wt. \%) } \\
\hline Quartz & 12.98 & 9.35 & 14.37 & 10.84 & 10.50 & 0.00 & 0.00 & 20.58 & 11.48 & 13.23 \\
\hline Corundum & 0.00 & 0.00 & 0.00 & 0.00 & 0.00 & 0.00 & 0.00 & 4.21 & 0.00 & 0.27 \\
\hline Orthoclase & 18.48 & 11.95 & 11.99 & 11.48 & 12.46 & 9.62 & 9.01 & 10.17 & 13.77 & 13.94 \\
\hline Albite & 26.46 & 32.51 & 30.04 & 28.55 & 29.73 & 39.61 & 25.97 & 22.82 & 20.07 & 28.32 \\
\hline Anorthite & 16.64 & 25.06 & 24.57 & 28.78 & 25.94 & 26.39 & 32.66 & 19.41 & 25.90 & 24.40 \\
\hline Diopside & 8.28 & 2.29 & 2.46 & 1.24 & 2.98 & 10.40 & 1.53 & 0.00 & 1.21 & 0.00 \\
\hline Hypersthene & 12.58 & 12.73 & 10.92 & 13.32 & 12.32 & 2.20 & 24.49 & 19.49 & 23.14 & 16.24 \\
\hline Olivine & 0.00 & 0.00 & 0.00 & 0.00 & 0.00 & 3.25 & 2.04 & 0.00 & 0.00 & 0.00 \\
\hline Magnetite & 3.07 & 4.46 & 4.10 & 4.26 & 4.28 & 3.88 & 2.07 & 1.49 & 2.80 & 2.08 \\
\hline Ilmenite & 1.11 & 1.23 & 1.18 & 1.13 & 1.33 & 1.37 & 1.80 & 1.46 & 1.30 & 1.19 \\
\hline Hematite & 0.00 & 0.00 & 0.00 & 0.00 & 0.00 & 2.68 & 0.00 & 0.00 & 0.00 & 0.00 \\
\hline Apatite & 0.40 & 0.42 & 0.38 & 0.40 & 0.44 & 0.59 & 0.44 & 0.36 & 0.34 & 0.33 \\
\hline \multicolumn{11}{|c|}{ TRACE ELEMENTS (ppm) } \\
\hline $\mathrm{Ba}$ & 368 & 306 & 280 & 368 & 338 & 181 & 231 & 262 & 502 & 430 \\
\hline$R b$ & 112 & 49 & 69 & 65 & 57 & 81 & 39 & 36 & 81 & 77 \\
\hline $\mathrm{Sr}$ & 193 & 301 & 230 & 314 & 316 & 314 & 327 & 264 & 298 & 307 \\
\hline $\mathrm{Y}$ & & 21 & & 18 & 23 & 40 & 28 & 24 & 23 & 22 \\
\hline $\mathrm{Z}_{x}$ & & 125 & & 75 & 133 & 68 & 71 & 111 & 137 & 142 \\
\hline $\mathrm{Nb}$ & & 8 & & 6 & 8 & 8 & 6 & 5 & 9 & 9 \\
\hline $\mathrm{Cu}$ & 69 & 22 & 21 & 18 & 29 & 359 & 72 & 47 & 33 & 5 \\
\hline $\mathrm{Pb}$ & 12 & 6 & 9 & 3 & 10 & 9 & $<2$ & $<2$ & 10 & 10 \\
\hline $\mathrm{Zn}$ & 71 & 78 & 65 & 88 & 90 & 81 & 118 & 80 & 93 & 86 \\
\hline $\mathrm{Ni}$ & & 11 & & 13 & 9 & 23 & 16 & 9 & 32 & 19 \\
\hline $\mathrm{Cr}$ & & 34 & & 35 & 24 & 24 & 18 & 23 & 85 & 35 \\
\hline V & & 182 & & 185 & 204 & 228 & 299 & 168 & 188 & 142 \\
\hline $\mathrm{Ga}$ & & 16 & & 15 & 19 & 20 & 22 & 13 & 15 & 18 \\
\hline Th & & $<2$ & & $<2$ & 2 & $<2$ & $<2$ & $<2$ & 10 & 10 \\
\hline As & 6.0 & 6.0 & 4.0 & 5.2 & 9.0 & 3.5 & & & & \\
\hline $\mathrm{F}$ & 430 & 410 & 490 & 460 & 460 & 560 & & & & \\
\hline Mo & 1.7 & 1.1 & 1.4 & 1.2 & 1.5 & 1.8 & & & & \\
\hline$S$ & 50 & 50 & 130 & 40 & 20 & 10 & & & & \\
\hline Sn & 2.6 & 2.0 & 1.8 & 1.3 & 1.5 & 2.2 & & & & \\
\hline $\mathrm{U}$ & 1.5 & 0.7 & 0.7 & 0.7 & 0.4 & 1.5 & & & & \\
\hline $\mathrm{Bi}$ & 0.07 & 0.06 & 0.06 & 0.06 & 0.05 & 0.09 & & & & \\
\hline $\mathrm{Li}$ & 22 & 19 & 19 & 26 & 17 & 19 & & & & \\
\hline W & nd & nd & nd & nd & nd & & & & & \\
\hline
\end{tabular}


Table 4. Chemical anaylses and CIPW normative mineralogy of samples from granodiorite-tonalite and granodiorite-monzogranite units.

\begin{tabular}{|c|c|c|c|c|c|c|}
\hline \multirow[b]{2}{*}{ SAMPLE } & \multicolumn{4}{|c|}{ GRANODIORITE-TONALITE } & \multicolumn{2}{|c|}{$\begin{array}{l}\text { GRANODIORITE- } \\
\text { MONZOGRANITE }\end{array}$} \\
\hline & $\begin{array}{l}\text { F11- } \\
1011\end{array}$ & $\begin{array}{l}\text { F11- } \\
1021\end{array}$ & $\begin{array}{l}\text { F11- } \\
1038\end{array}$ & $\begin{array}{l}\text { F11- } \\
1078\end{array}$ & $\begin{array}{l}\text { F14- } \\
1210\end{array}$ & $\begin{array}{l}\text { F14- } \\
1211\end{array}$ \\
\hline \multicolumn{7}{|c|}{ MAJOR OXIDES (wt. \%) } \\
\hline $\mathrm{SiO}_{2}$ & 67.00 & 67.90 & 69.80 & 67.95 & 67.00 & 68.50 \\
\hline $\mathrm{TiO}_{2}$ & 0.50 & 0.40 & 0.40 & 0.48 & 0.43 & 0.40 \\
\hline $\mathrm{Al}_{2} \mathrm{O}_{3}$ & 15.33 & 14.9 & 15.7 & 15.13 & 15.7 & 14.70 \\
\hline $\mathrm{Fe}_{2} \mathrm{O}_{3}$ & 0.84 & 1.52 & 1.67 & 0.82 & 1.63 & 1.24 \\
\hline $\mathrm{FeO}$ & 2.76 & 1.78 & 1.38 & 2.70 & 2.13 & 1.85 \\
\hline $\mathrm{MnO}$ & 0.10 & 0.06 & 0.08 & 0.17 & 0.10 & 0.09 \\
\hline $\mathrm{MgO}$ & 1.91 & 1.10 & 0.91 & 1.96 & 1.60 & 1.40 \\
\hline $\mathrm{CaO}$ & 1.46 & 2.60 & 2.90 & 1.62 & 2.10 & 1.70 \\
\hline $\mathrm{Na}_{2} \mathrm{O}$ & 4.95 & 4.9 & 4.7 & 4.53 & 3.4 & 3.0 \\
\hline $\mathrm{K}_{2} \mathrm{O}$ & 1.98 & 2.0 & 2.4 & 1.84 & 3.5 & 3.9 \\
\hline $\mathrm{P}_{2} \mathrm{O}_{5}$ & 0.12 & 0.15 & 0.14 & 0.12 & 0.20 & 0.17 \\
\hline LOI & 2.0 & 2.1 & 1.1 & 2.5 & 1.4 & 1.3 \\
\hline TOTAL OXIDES & 98.95 & 99.41 & 101.18 & 99.82 & 99.19 & 98.25 \\
\hline \multicolumn{7}{|c|}{ NORMATIVE MINERALOGY (wt. \%) } \\
\hline Quartz & 24.03 & 24.97 & 25.79 & 27.51 & 27.18 & 30.97 \\
\hline Corundum & 2.76 & 0.32 & 0.43 & 3.11 & 3.05 & 2.95 \\
\hline Orthoclase & 12.07 & 12.14 & 14.17 & 11.17 & 21.15 & 23.77 \\
\hline Albite & 43.20 & 42.60 & 39.73 & 39.38 & 29.42 & 26.18 \\
\hline Anorthite & 6.66 & 12.25 & 13.46 & 7.45 & 9.32 & 7.55 \\
\hline Diopside & 0.00 & 0.00 & 0.00 & 0.00 & 0.00 & 0.00 \\
\hline Hypersthene & 8.76 & 4.32 & 2.91 & 8.92 & 6.16 & 5.53 \\
\hline Olivine & 0.00 & 0.00 & 0.00 & 0.00 & 0.00 & 0.00 \\
\hline Magnetite & 1.26 & 2.26 & 2.42 & 1.22 & 2.42 & 1.85 \\
\hline Ilmenite & 0.98 & 0.78 & 0.76 & 0.94 & 0.84 & 0.78 \\
\hline Hematite & 0.00 & 0.00 & 0.00 & 0.00 & 0.00 & 0.00 \\
\hline Apatite & 0.29 & 0.36 & 0.32 & 0.29 & 0.47 & 0.41 \\
\hline \multicolumn{7}{|c|}{ TRACE ELEMENTS (ppm) } \\
\hline $\mathrm{Ba}$ & 640 & 395 & 499 & 365 & 354 & 350 \\
\hline $\mathrm{Rb}$ & 79 & 64 & 83 & 84 & 128 & 147 \\
\hline $\mathrm{Sr}$ & 161 & 211 & 309 & 126 & 273 & 255 \\
\hline $\mathrm{Y}$ & 29 & 20 & 19 & 20 & 28 & 22 \\
\hline $\mathrm{Zr}$ & 166 & 142 & 189 & 151 & 200 & 166 \\
\hline $\mathrm{Nb}$ & 9 & 9 & 9 & 7 & 13 & 13 \\
\hline $\mathrm{Cu}$ & $<2$ & $<2$ & 5 & 3 & 18 & 3 \\
\hline $\mathrm{Pb}$ & 35 & 11 & 11 & 10 & 12 & 13 \\
\hline $\mathrm{Zn}$ & 181 & 42 & 48 & 140 & 69 & 61 \\
\hline $\mathrm{Ni}$ & 3 & 8 & 9 & 5 & 15 & 16 \\
\hline $\mathrm{Cr}$ & 5 & 24 & 21 & 3 & 29 & 31 \\
\hline $\mathrm{V}$ & 56 & 74 & 44 & 63 & 73 & 72 \\
\hline $\mathrm{Ga}$ & 17 & 17 & 16 & 15 & 20 & 14 \\
\hline Th & 6 & 8 & 6 & 4 & 16 & 17 \\
\hline As & & 0.5 & 2.70 & & 4.0 & 9.0 \\
\hline $\mathrm{F}$ & & 390 & 460 & & 490 & 430 \\
\hline Mo & & 1.3 & 1.2 & & 1.5 & 1.8 \\
\hline $\mathrm{S}$ & & & & & 50 & \\
\hline $\mathrm{Sn}$ & & 3.0 & 3.3 & & 1.8 & 2.1 \\
\hline $\mathrm{U}$ & & 1.8 & 2.0 & & 3.6 & 3.9 \\
\hline $\mathrm{Bi}$ & & 0.05 & 0.05 & & 0.07 & 0.07 \\
\hline $\mathrm{Li}$ & & 6 & 15 & & 39 & 39 \\
\hline
\end{tabular}


Table 5. Chemical analyses and CIPW normative mineralogy of samples from the coarse-grained monzogranite unit.

$\begin{array}{lllllllllllll}\text { SAMPLE } & \text { F14- } & \text { F14- } & \text { F14- } & \text { F14- } & \text { F14- } & \text { F14- } & \text { F11- } & \text { F11- } & \text { F11- } & \text { F11- } & \text { CH-87 } & \text { CH-87 } \\ & 1078 & 1098 & 1099 & 1143 & 1158 & 1224 & 1016 & 1041 & 1042 & 1051 & 016 & 027\end{array}$

\begin{tabular}{|c|c|c|c|c|c|c|c|c|c|c|c|c|}
\hline \multicolumn{13}{|c|}{ MAJOR OXIDES (wt. \%) } \\
\hline $\mathrm{SiO}_{2}$ & 73.20 & 71.00 & 73.00 & 75.50 & 71.80 & 73.80 & 73.00 & 75.40 & 73.80 & 75.90 & 73.75 & 73.69 \\
\hline $\mathrm{TiO}_{2}$ & 0.22 & 0.32 & 0.24 & 0.19 & 0.48 & 0.21 & 0.21 & 0.22 & 0.22 & 0.21 & 0.19 & 0.19 \\
\hline $\mathrm{Al}_{2} \mathrm{O}_{3}$ & 13.6 & 13.6 & 13.2 & 12.3 & 13.9 & 13.5 & 13.3 & 13.2 & 13.2 & 13.1 & 13.56 & 13.65 \\
\hline $\mathrm{Fe}_{2} \mathrm{O}_{3}$ & 0.57 & 0.74 & 0.54 & 0.27 & 0.90 & 0.84 & 0.64 & 0.69 & 0.51 & 0.77 & 0.25 & 0.26 \\
\hline $\mathrm{EO}$ & 1.65 & 1.85 & 1.58 & 1.11 & 2.34 & 1.31 & 1.49 & 1.45 & 1.34 & 1.29 & 1.52 & 1.61 \\
\hline InO & 0.05 & 0.05 & 0.06 & 0.04 & 0.07 & 0.0 & 0.05 & $0 .($ & 0.04 & 0. & 0.04 & 0.06 \\
\hline IgO & 0.33 & 0.69 & 0.33 & 0.20 & 0.5 & 0.24 & 0.20 & 0.20 & 0.20 & 0. & 0.79 & 2.09 \\
\hline $\mathrm{aO}$ & 1.00 & 1.00 & 0.93 & 0.46 & 1.70 & 0.58 & 0.88 & 0.55 & 0.90 & 0. & 0.18 & 0.43 \\
\hline $\mathrm{Na}_{2} \mathrm{O}$ & 3.9 & 3.8 & 3.8 & 3.4 & 2.9 & 3.8 & 4.0 & 3.9 & 3.6 & 3. & 3.63 & 3.10 \\
\hline $\mathrm{K}_{2} \mathrm{O}$ & 4.6 & 4.1 & 4.3 & 4.7 & 4.5 & 4.3 & 4.0 & 4.4 & 4.0 & 4.0 & 4.23 & 4.33 \\
\hline $\mathrm{P}_{2} \mathrm{O}_{5}$ & 0.06 & 0.09 & 0.09 & 0.06 & 0.14 & 0.03 & 0.04 & 0.05 & 0.02 & 0.04 & 0.04 & 0.04 \\
\hline LOI & 1.2 & 1.5 & 1.5 & 0.74 & 0.85 & 0.71 & 1.0 & 0.07 & 1.3 & 0.73 & 0.90 & 0.90 \\
\hline TOTAL O & 100.38 & 98.74 & 99.5 & 98.70 & 100.13 & 9938 & 98.81 & 100.18 & 99.13 & 100.78 & 99.10 & 100.35 \\
\hline & & & & & & & & & & & & \\
\hline Quartz & 29.65 & 30.05 & 31.84 & 36.55 & 32.56 & 3.5 & 2.17 & 33.68 & 35.36 & 35.8 & 34.56 & 34.20 \\
\hline$C$ & & & & & & & & & & & & 3.20 \\
\hline Drth & תח & 24.91 & 25 & 8.27 & 26. & 5 & 6 & 25.97 & 24 & 23. & 25 & 25.73 \\
\hline Albi & 33.27 & 33.06 & 32.78 & 29.29 & 24.71 & 32.58 & 34.60 & 32.96 & 31.13 & 32.98 & 31.28 & 26.37 \\
\hline Anorthite & 4.61 & 4.50 & 4.10 & 1.92 & 7.57 & 2.72 & 4.20 & 2.40 & 4.43 & 2.42 & & 1.88 \\
\hline & & & & & & 0. & 0.00 & 0. & & & & 0.00 \\
\hline & & & & & 10 & 1 & 25 & 23 & 2 & & 4 & 7.79 \\
\hline Ol & & & & & & & 0.00 & 0. & 0.00 & 0.00 & 0.00 & 0.00 \\
\hline Magnetite & 0.83 & 1.10 & 0.80 & 0.40 & 1.31 & 1.24 & 0.95 & 1.00 & 0.76 & 1.12 & 0.37 & 0.38 \\
\hline Ilmenite & 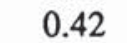 & 0.63 & & 0.3 & 0.9 & 0.40 & 0.41 & 0.42 & 0.43 & & & 0.36 \\
\hline Hema & & 0.0 & 0. & 0. & 0. & 0. & 0.0 & 0.0 & $0 .(\mathrm{r})$ & 0. & 0.00 & 0.00 \\
\hline Apatite & 0.14 & 0.21 & 0.21 & 0.14 & 0.33 & 0.0 & 0.0 & 0.1 & 0.05 & 0.09 & 0.09 & 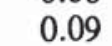 \\
\hline
\end{tabular}

TRACE ELEMENTS (ppm)

\begin{tabular}{|c|c|c|c|c|c|c|c|c|c|c|c|c|}
\hline $\mathrm{Ba}$ & 550 & 613 & 587 & 688 & 596 & 530 & 552 & 491 & 526 & 431 & 513 & 582 \\
\hline $\mathrm{Rb}$ & 132 & 142 & 144 & 130 & 183 & 127 & 144 & 148 & 144 & 120 & 143 & 151 \\
\hline $\mathrm{Sr}$ & 86 & 119 & 101 & 64 & 112 & 97 & 97 & 74 & 59 & 74 & 59 & 98 \\
\hline Y & & 34 & 31 & 32 & 63 & & 37 & 36 & 29 & 27 & 38 & 36 \\
\hline $\mathrm{Zr}_{\mathrm{r}}$ & & 210 & 198 & 173 & 241 & & 191 & 183 & 191 & 207 & 204 & 196 \\
\hline $\mathrm{Nb}$ & & 14 & 13 & 14 & 16 & & 15 & 14 & 14 & 17 & 15 & 12 \\
\hline $\mathrm{Cu}$ & 7 & 2 & $<2$ & 4 & 2 & 3 & 1 & $<2$ & $<2$ & $<2$ & 1 & 9 \\
\hline $\mathrm{Pb}$ & 12 & 14 & 22 & 13 & 36 & 14 & 13 & 12 & 11 & 14 & $<2$ & 3 \\
\hline $\mathrm{Zn}$ & 31 & 37 & 58 & 33 & 84 & 41 & 42 & 37 & 35 & 34 & 32 & 40 \\
\hline $\mathrm{Ni}$ & & 19 & 15 & 14 & 31 & & 18 & 20 & 16 & 10 & 3 & 5 \\
\hline $\mathrm{Cr}_{\mathrm{r}}$ & & 24 & 24 & 19 & 20 & & 29 & 24 & 21 & 20 & 1 & 5 \\
\hline V & & 29 & 13 & 10 & 27 & & 10 & 4 & 7 & 6 & 3 & 5 \\
\hline $\mathrm{Ga}$ & & 17 & 17 & 12 & 21 & & 19 & 14 & 14 & 18 & 16 & 15 \\
\hline Th & & 12 & 13 & 17 & 12 & & 15 & 11 & 12 & 10 & 15 & 4 \\
\hline As & 4.0 & 4.5 & 5.2 & 6.0 & 1.0 & 2.7 & 10.0 & 2.7 & 4.0 & 68.5 & & \\
\hline$F$ & 490 & 310 & 360 & 200 & 950 & 310 & 280 & 340 & 180 & 260 & & \\
\hline Mo & 1.3 & 1.5 & 2.0 & 1.5 & 2.2 & 1.3 & 1.5 & 1.5 & 1.2 & 2.1 & & \\
\hline S & & & & & & & 20 & & 30 & & & \\
\hline Sn & 2.7 & 4.0 & 3.2 & 3.0 & 5.1 & 3.1 & 6.5 & 4.2 & 4.7 & 3.4 & & \\
\hline$U$ & 2.1 & 1.6 & 2.9 & 2.7 & 2.6 & 3.3 & 2.8 & 2.9 & 2.7 & 2.0 & & \\
\hline $\mathrm{Bi}$ & 0.04 & 0.05 & 0.09 & 0.41 & 0.06 & 0.05 & 0.05 & 0.05 & 0.05 & 0.07 & & \\
\hline $\mathrm{Li}$ & 11 & 17 & 16 & 11 & 26 & 11 & 8 & 7 & 6 & 41 & & \\
\hline
\end{tabular}


Table 6. Chemical analyses and CIPW normative mineralogies of samples from the fine-grained monzogranite unit and aplite dykes.

\begin{tabular}{|c|c|c|c|c|c|c|c|c|}
\hline \multirow[b]{2}{*}{ SAMPLE } & \multicolumn{5}{|c|}{ FINE-GRAINED MONZOGRANITE } & \multicolumn{3}{|c|}{ APLITE } \\
\hline & $\begin{array}{l}\text { F14- } \\
1103\end{array}$ & $\begin{array}{l}\text { F14- } \\
1107\end{array}$ & $\begin{array}{l}\text { F14- } \\
1117\end{array}$ & $\begin{array}{l}\text { F14- } \\
1118\end{array}$ & $\begin{array}{l}\text { F14- } \\
1134\end{array}$ & $\begin{array}{l}\text { F14- } \\
1029\end{array}$ & $\begin{array}{l}\text { F14- } \\
1041\end{array}$ & $\begin{array}{c}\mathrm{CH}-87 \\
024\end{array}$ \\
\hline \multicolumn{9}{|c|}{ MAJOR OXIDES (wt. \%) } \\
\hline $\mathrm{SiO}_{2}$ & 73.00 & 71.80 & 71.30 & 71.00 & 71.20 & 75.00 & 78.20 & 76.88 \\
\hline $\mathrm{TiO}_{2}$ & 0.16 & 0.40 & 0.37 & 0.38 & 0.34 & 0.10 & 0.13 & 0.06 \\
\hline $\mathrm{Al}_{2} \mathrm{O}_{3}$ & 13.1 & 14.5 & 14.0 & 14.0 & 14.0 & 12.3 & 11.8 & 12.84 \\
\hline $\mathrm{Fe}_{2} \mathrm{O}_{3}$ & 0.31 & 0.91 & 0.66 & 0.59 & 0.56 & 0.51 & 1.06 & 0.11 \\
\hline $\mathrm{FeO}$ & 1.16 & 2.06 & 1.93 & 1.99 & 1.84 & 0.30 & 0.49 & 0.71 \\
\hline $\mathrm{MnO}$ & 0.03 & 0.06 & 0.06 & 0.06 & 0.05 & 0.01 & 0.02 & 0.03 \\
\hline $\mathrm{MgO}$ & 0.23 & 0.96 & 0.80 & 0.79 & 0.71 & 0.08 & 0.13 & 0.69 \\
\hline $\mathrm{CaO}$ & 0.88 & 2.10 & 1.80 & 1.70 & 2.00 & 0.29 & 0.68 & 0.17 \\
\hline $\mathrm{Na}_{2} \mathrm{O}$ & 3.3 & 3.0 & 3.1 & 3.0 & 2.9 & 2.8 & 2.4 & 3.27 \\
\hline $\mathrm{K}_{2} \mathrm{O}$ & 4.9 & 4.2 & 4.2 & 4.1 & 3.9 & 6.3 & 6.1 & 4.52 \\
\hline $\mathrm{P}_{2} \mathrm{O}_{5}$ & 0.04 & 0.09 & 0.15 & 0.09 & 0.09 & 0.02 & 0.04 & 0.02 \\
\hline LOI & 0.87 & 1.1 & 1.1 & 1.1 & 0.86 & 0.5 & 0.6 & 0.5 \\
\hline TOTAL OXIDES & 97.98 & 101.18 & 99.47 & 98.80 & 98.45 & 98.21 & 101.65 & 99.80 \\
\hline \multicolumn{9}{|c|}{ NORMATIVE MINERALOGY (wt. \%) } \\
\hline Quartz & 33.14 & 31.45 & 31.95 & 32.84 & 34.02 & 34.72 & 38.95 & 38.97 \\
\hline Corundum & 0.89 & 1.42 & 1.46 & 1.79 & 1.63 & 0.40 & 0.11 & 2.32 \\
\hline Orthoclase & 29.81 & 24.80 & 25.23 & 24.80 & 23.61 & 38.10 & 35.67 & 26.90 \\
\hline Albite & 28.75 & 25.36 & 26.66 & 25.98 & 25.14 & 24.25 & 20.09 & 27.86 \\
\hline Anorthite & 4.23 & 9.82 & 8.08 & 8.03 & 9.56 & 1.34 & 3.08 & 0.72 \\
\hline Diopside & 0.00 & 0.00 & 0.00 & 0.00 & 0.00 & 0.00 & 0.00 & 0.00 \\
\hline Hypersthene & 2.30 & 4.87 & 4.57 & 4.73 & 4.32 & 0.20 & 0.32 & 2.91 \\
\hline Olivine & 0.00 & 0.00 & 0.00 & 0.00 & 0.00 & 0.00 & 0.00 & 0.00 \\
\hline Magnetite & 0.46 & 1.32 & 0.97 & 0.88 & 0.83 & 0.73 & 1.25 & 0.16 \\
\hline Ilmenite & 0.31 & 0.76 & 0.71 & 0.74 & 0.66 & 0.19 & 0.24 & 0.11 \\
\hline Hematite & 0.00 & 0.00 & 0.00 & 0.00 & 0.00 & 0.02 & 0.18 & 0.00 \\
\hline Apatite & 0.10 & 0.21 & 0.35 & 0.21 & 0.21 & 0.05 & 0.09 & 0.05 \\
\hline \multicolumn{9}{|c|}{ TRACE ELEMENTS (ppm) } \\
\hline $\mathrm{Ba}$ & 410 & 504 & 512 & 527 & 500 & 142 & 265 & 64 \\
\hline $\mathrm{Rb}$ & 178 & 146 & 152 & 156 & 151 & 327 & 282 & 266 \\
\hline $\mathrm{Sr}$ & 93 & 146 & 145 & 151 & 141 & 23 & 51 & 18 \\
\hline $\mathrm{Y}$ & & 29 & 33 & 33 & & & & 44 \\
\hline $\mathrm{Zr}$ & & 191 & 187 & 194 & & & & 87 \\
\hline $\mathrm{Nb}$ & & 12 & 12 & 11 & & & & 18 \\
\hline $\mathrm{Cu}$ & 33 & 5 & 1 & 6 & 14 & 6 & 7 & $<2$ \\
\hline $\mathrm{Pb}$ & 38 & 21 & 22 & 21 & 10 & 18 & 16 & 23 \\
\hline $\mathrm{Zn}$ & 73 & 39 & 49 & 44 & 32 & 5 & 15 & 20 \\
\hline $\mathrm{Ni}$ & & 18 & 19 & 21 & & & & 2 \\
\hline $\mathrm{Cr}$ & & 37 & 32 & 30 & & & & 4 \\
\hline $\mathrm{v}$ & & 52 & 49 & 46 & & & & 2 \\
\hline $\mathrm{Ga}$ & & 17 & 16 & 14 & & & & 18 \\
\hline $\mathrm{Th}$ & & 14 & 13 & 14 & & & & 57 \\
\hline As & 4.0 & 4.0 & 3.5 & 5.2 & 76.5 & 4.0 & 4.0 & \\
\hline $\mathrm{F}$ & 390 & 520 & 640 & 520 & 460 & 640 & 170 & \\
\hline Mo & 2.2 & 1.4 & 1.9 & 1.5 & 1.9 & 1.8 & 1.9 & \\
\hline S & 980 & 310 & 20 & & 380 & & 20 & \\
\hline Sn & 2.5 & 2.9 & 4.4 & 3.3 & 3.7 & 2.2 & 3.2 & \\
\hline $\mathrm{U}$ & 6.7 & 4.2 & 3.2 & 2.9 & 4.4 & 7.3 & 4.7 & \\
\hline $\mathrm{Bi}$ & 0.32 & 0.05 & 0.06 & 0.06 & 1.50 & 0.10 & 0.07 & \\
\hline $\mathrm{Li}$ & 11 & 40 & 36 & 35 & 31 & 1 & 6 & \\
\hline
\end{tabular}


Table 7. Rare-earth element data* (in ppm) from the Creignish Hills Pluton.

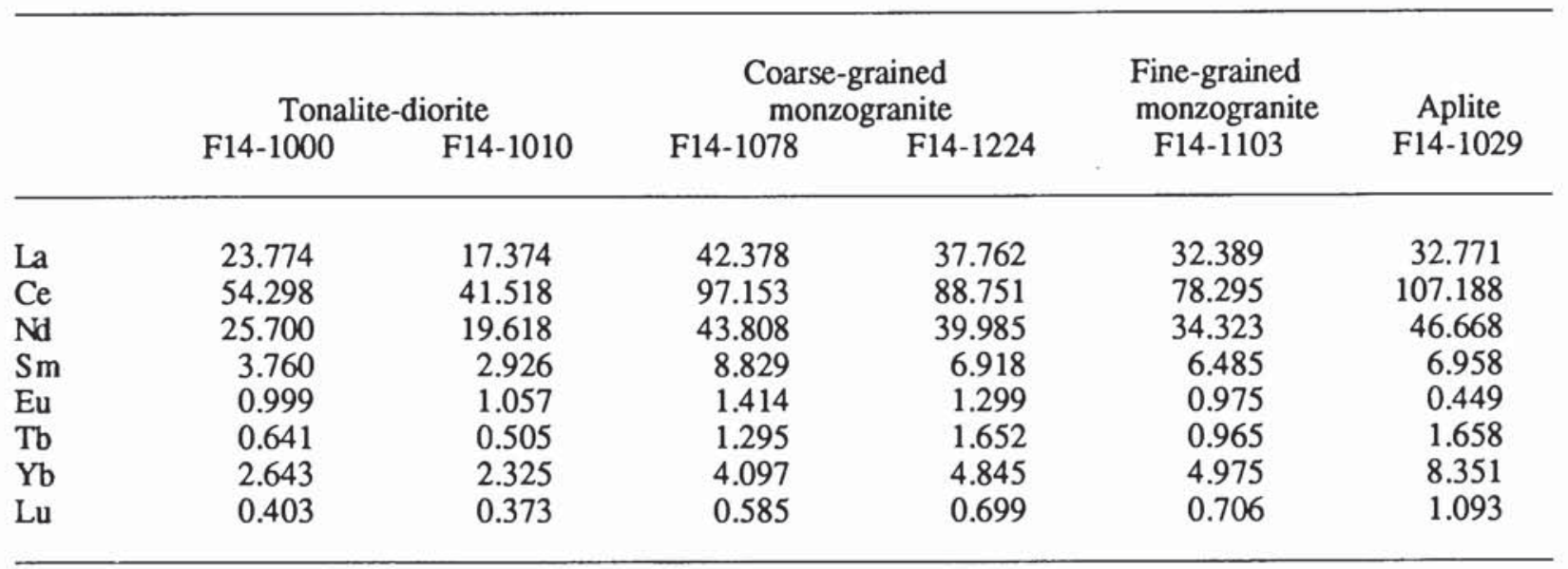

*See Appendix A for method of analyses.

The granodiorite-monzogranite unit (Table 4) has $\mathrm{SiO}_{2}$ contents very similar to those in the granodiorite-tonalite unit, and overall these two units are chemically similar, except that the analyzed samples from the granodiorite-monzogranite unit have lower $\mathrm{Na}_{2} \mathrm{O}$ and higher $\mathrm{K}_{2} \mathrm{O}, \mathrm{Rb}, \mathrm{Nb}, \mathrm{Ni}, \mathrm{Cr}$, and Th.

Samples from the coarse-grained monzogranite unit range in $\mathrm{SiO}_{2}$ content from about 71 to $75 \%$ (Table 5). $\mathrm{Al}_{2} \mathrm{O}_{3}, \mathrm{Fe}_{2} \mathrm{O}_{3} \mathrm{~T}$, $\mathrm{MgO}, \mathrm{MnO}, \mathrm{CaO}, \mathrm{Ba}, \mathrm{Sr}, \mathrm{Ni}, \mathrm{V}$, and $\mathrm{Ga}$ show strong negative correlation with $\mathrm{SiO}_{2}$ (e.g., Figs. 3, 4).

The fine-grained monzogranite ranges in $\mathrm{SiO}_{2}$ content from 71 to $73 \%$ (Table 6), with an average of $71.7 \%$, significantly lower than the average of $73.7 \%$ for the coarse-grained monzogranite. Compared to the coarse-grained monzogranite, the fine-grained monzogranite has higher $\mathrm{TiO}_{2}, \mathrm{Al}_{2} \mathrm{O}_{3}, \mathrm{Fe}_{2} \mathrm{O}_{3}, \mathrm{MnO}$, $\mathrm{MgO}, \mathrm{CaO}$, and $\mathrm{Sr}$.

Samples from aplitic dykes (Table 6) were analyzed for comparison with the major units of the pluton. They are high in silica (average 76.7\%), and correspondingly low in most other major elements $\left(\mathrm{TiO}_{2}, \mathrm{Al}_{2} \mathrm{O}_{3}, \mathrm{Fe}_{2} \mathrm{O}_{3} \mathrm{~T}, \mathrm{MgO}, \mathrm{MnO}, \mathrm{CaO}\right.$, and $\mathrm{P}_{2} \mathrm{O}_{5}$ ) except $\mathrm{K}_{2} \mathrm{O}$. The aplites are also high in $\mathrm{Rb}$ and $\mathrm{Th}$, and low in $\mathrm{Ba}, \mathrm{Sr}, \mathrm{Zr}, \mathrm{Zn}, \mathrm{Ni}$, and $\mathrm{Cr}$.

Six samples analyzed for rare-earth elements (Table 7) all show significant enrichment in light REE compared to heavy REE (Fig. 5). The two samples from the tonalite-diorite unit have lower total REE abundances than samples from the other units, and minor Eu anomalies. The aplite has the highest total REE abundance and a very strong negative Eu anomaly. Patterns in the monzogranitic samples are similar to one another, with the lower silica fine-grained monzogranite sample having generally lower abundances of REE.

\section{AGE}

A minimum age for the tonalite-diorite unit is provided by ${ }^{40} \mathrm{Ar} /{ }^{\beta 9} \mathrm{Ar}$ cooling ages of about $540 \mathrm{Ma}$ for hornblendes from the unit (Keppie et al., 1989). These ages are similar to cooling ages obtained from several other dioritic to granodioritic plutons in the Bras d'Or Terrane of central Cape Breton Island that are consid- ered to have crystallized between 555-565 Ma on the basis of U$\mathrm{Pb}$ dating (Keppie et al., 1989; Dunning et al., 1989; Barr et al., in press). Plutons of this age are characteristic of the Bras d'Or Terrane.

$\mathrm{A} \mathrm{Rb}-\mathrm{Sr}$ whole-rock isochron for the Creignish Hills Pluton, using samples from the tonalite-diorite unit, coarse- and finegrained monzogranite units, and aplite dykes, indicates an age of $446 \pm 13 \mathrm{Ma}$ with an initial ${ }^{87} \mathrm{Sr} /{ }^{\beta 6} \mathrm{Sr}$ ratio of $0.7156 \pm 0.0033$ (Table 8, Fig.6). This age is not consistent with the ${ }^{40} \mathrm{Ar} /{ }^{99} \mathrm{Ar}$ age of the tonalite-diorite unit. Exclusion of the two tonalite-diorite samples from the age calculation results in little change in the apparent age (441 $\pm 8 \mathrm{Ma})$. However, this age is strongly controlled by the two aplite samples. If the aplites are omitted, the six remaining samples yield a poorly constrained age of 473 $\pm 102 \mathrm{Ma}$.

We interpret these data to indicate that the tonalite-diorite unit, with a minimum age of ca. $540 \mathrm{Ma}$, is significantly older than the monzogranitic units. This is further supported by the similarity of the coarse-grained monzogranite to monzogranites of the Kellys Mountain and Cape Smoky plutons (Fig. 7) that have yielded U-Pb ages of about $495 \mathrm{Ma}$ (Dunning et al., 1989; Barr et al., in press). Hence, we suggest that the monzogranitic units of the Creignish Hills Pluton are also of approximately this age.

Older (ca. $560 \mathrm{Ma}$ ) dioritic rocks occur adjacent to the Cape Smoky granite (Dunning et al., 1989), and dioritic rocks associated with the Kellys Mountain granite may also be older and not co-genetic with the granite (Barr et al., in press; Barr et al., 1982). These dioritic units may be correlative with the tonalite-diorite unit of the Creignish Hills Pluton.

\section{PETROGENESIS}

If the age interpretations discussed above are correct, then the tonalite-diorite unit and the monzogranitic units represent separate unrelated magmas. The relationship of the intermediate granodiorite-tonalite and granodiorite-monzogranite units is not constrained by age data. However, the composition gaps (Figs. 

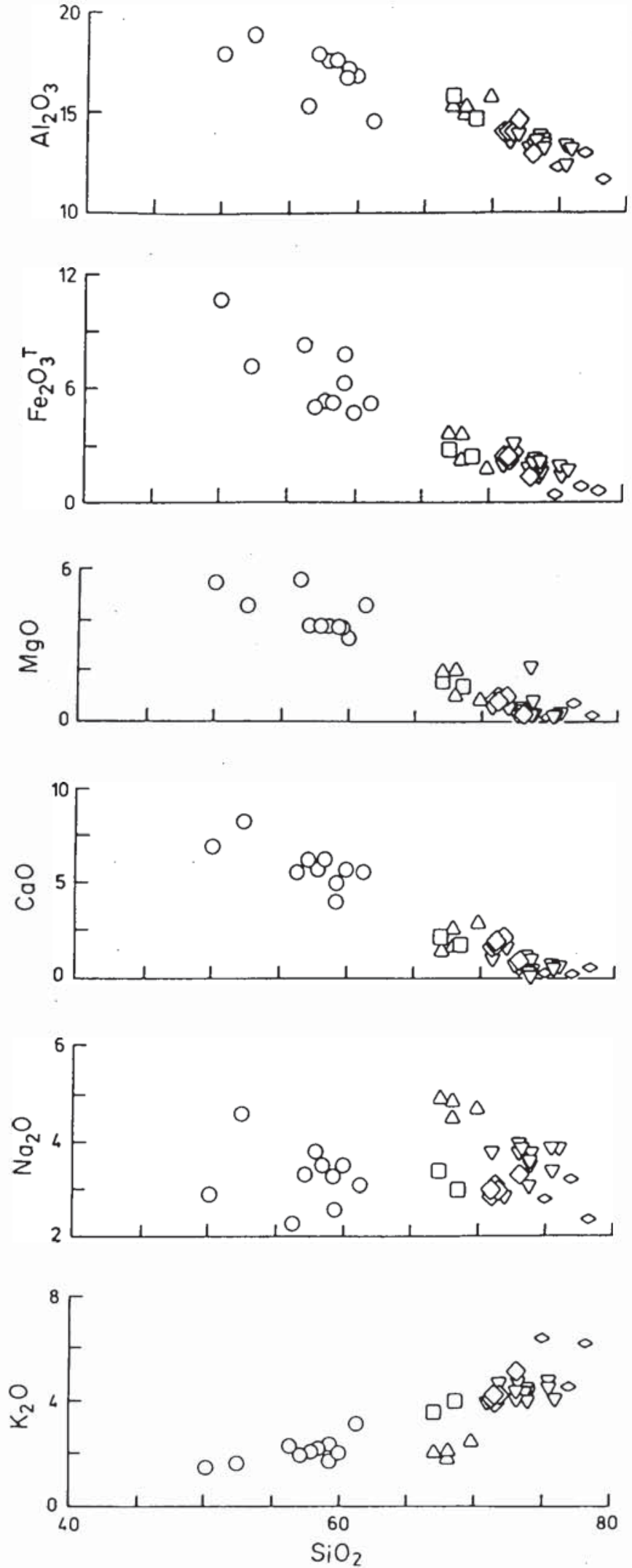

Fig. 3. Silica variation diagrams for selected major element oxides. Symbols as in Figure 2, except unshaded.

$3,4,8$ ) separating the tonalite-diorite samples from all the other units suggests that the intermediate units are co-genetic with the
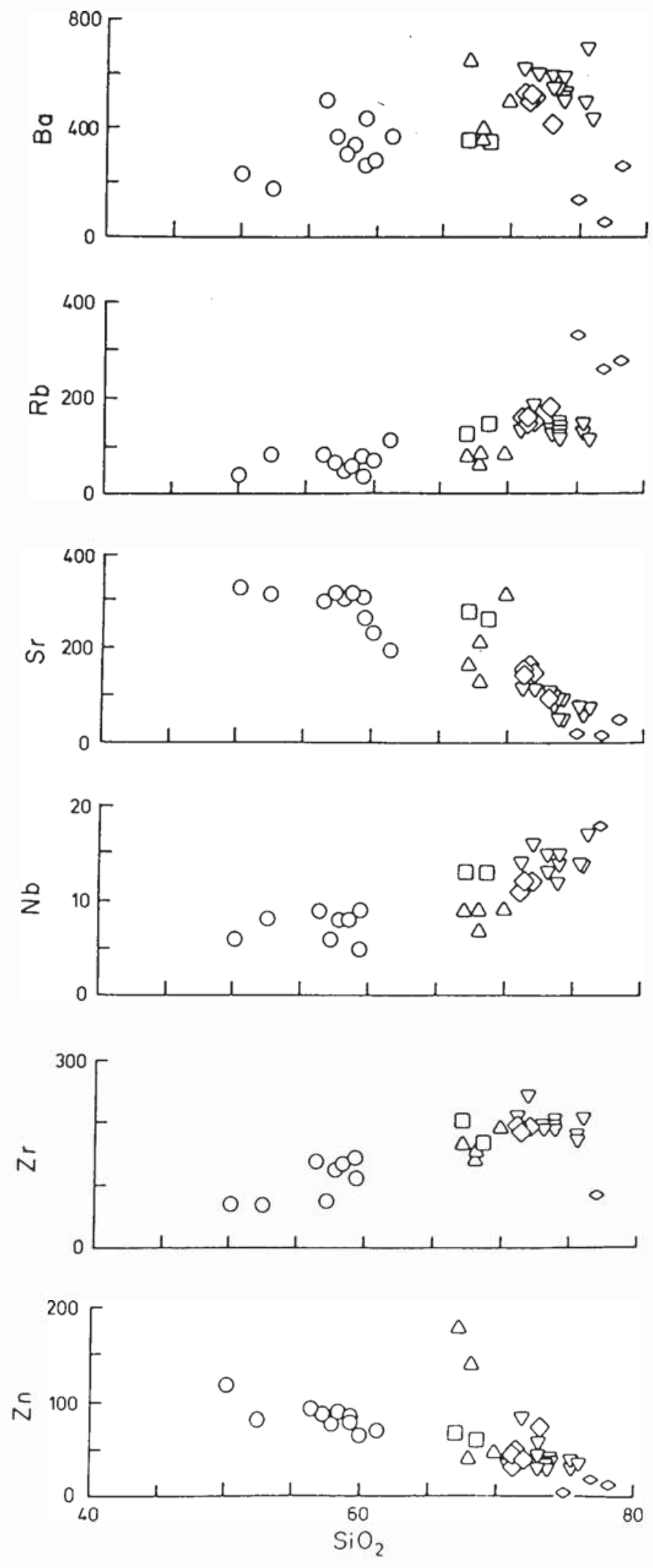

Fig.4. Silica variation diagrams for selected trace elements. Symbols as in Figure 2, except unshaded.

monzogranitic units. However, it is possible that none of these units has a co-genetic relationship with any other unit. 
Major element trends (Figs. 3,4) and plots of Rb-Sr and BaSr (Fig. 9a, b) suggest that hornblende fractionation ( \pm biotite accumulation) was the dominant process in the evolution of the tonalite-diorite unit, combined with some plagioclase fractionation. Plagioclase fractionation appears to have dominated evolution within each of the other units. The $\mathrm{Rb}-\mathrm{Ba}-\mathrm{Sr}$ data are

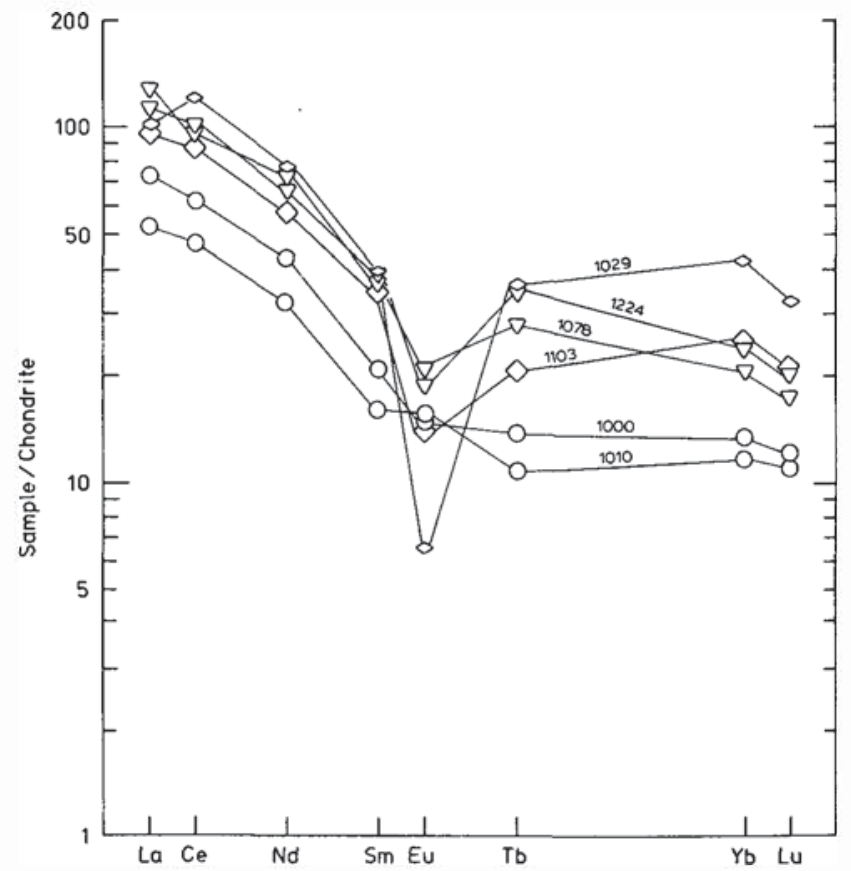

Fig. 5. Plot of chondrite-normalized rare-earth element data from Table 6. Chondrite normalizing values from Haskin et al. (1968). Sample prefixes all F14. supported by the REE data that indicate that the samples from the tonalite-diorite unit lack prominent Eu anomalies, and hence have probably not undergone significant feldspar fractionation (e.g., Hanson, 1980), whereas the other units all show large negative Eu anomalies consistent with extensive feldspar fractionation (Fig. 5).

The petrogenetic relationships among the intermediate and felsic units are not clear. The fine-grained monzogranite is younger than the coarse-grained monzogranite on the basis of field relations, but it appears to be less evolved chemically, and hence was probably not directly related to the coarse-grained monzogranite magma by fractional crystallization processes. Either of the monzogranitic units could be related to the granodiorite-tonalite and granodiorite-monzogranite units by fractional crystallization of feldspar and mafic minerals (Fig. 9).

The decrease in $\mathrm{Ba}$ in the aplite samples and the lack of change in $\mathrm{Rb}$ content are both compatible with $\mathrm{K}$-feldspar fractionation. The $\mathrm{Rb}-\mathrm{Sr}$ isotopic data (see section on Age) suggest that the aplitic dykes may be significantly younger than the rest of the pluton. Alternatively, their Rb-Sr isotopic system may have been disturbed subsequent to crystallization.

Overall, the petrologic characteristics of the Creignish Hills Pluton indicate that it is calc-alkalic (Fig. 8) and that it formed in an orogenic ("volcanic arc") setting (Fig. 10). Although the age of the pluton is not known with certainty, the tonalite-diorite unit probably formed as a result of late Precambrian to early Cambrian ( $565-555 \mathrm{Ma}$ ) subduction under the Bras d'Or Terrane. The tectonic setting for the ca. 495 Ma plutons in the Bras d'Or Terrane, with which the remainder of the pluton is tentatively correlated, is less clear. These plutons also have volcanic arc characteristics and are chemically similar (Fig. 7) to the average I-type granite of Whalen et al. (1987). However, it is unlikely that

Table 8. Rb-Sr isotopic data* from the Creignish Hills Pluton.

\begin{tabular}{|c|c|c|c|c|}
\hline Sample No. & $\mathrm{Rb}(\mathrm{ppm})$ & $\mathrm{Sr}(\mathrm{ppm})$ & ${ }^{87} \mathrm{Rb} /{ }^{86} \mathrm{Sr}$ & ${ }^{87} \mathrm{Sr} /{ }^{86} \mathrm{Sr}$ \\
\hline \multicolumn{5}{|c|}{ Aplite } \\
\hline F14-1029 & 297 & 21.7 & 40.5 & 0.9718 \\
\hline F14-1041 & 249 & 49.8 & 14.6 & 0.8087 \\
\hline \multicolumn{5}{|c|}{ Fine-grained monzogranite } \\
\hline F14-1103 & 199 & 77.7 & 7.46 & 0.7661 \\
\hline F14-1134 & 162 & 135 & 3.48 & 0.7383 \\
\hline \multicolumn{5}{|c|}{ Coarse-grained monzogranite } \\
\hline F11-1016 & 153 & $105^{\circ}$ & 4.24 & 0.7475 \\
\hline F11-1041 & 154 & 79.2 & 5.64 & 0.7535 \\
\hline F14-1078 & 163 & 110 & 4.30 & 0.7423 \\
\hline F14-1224 & 174 & 94.0 & 5.37 & 0.7496 \\
\hline \multicolumn{5}{|c|}{ Tonalite-diorite } \\
\hline F14-1000 & 122 & 273 & 1.30 & 0.7216 \\
\hline F14-1010 & 70.6 & 301 & 0.678 & 0.7133 \\
\hline
\end{tabular}

\footnotetext{
*Analyses by R.F. Cormier, Saint Francis Xavier University, Antigonish, Nova Scotia.
} Errors at $95 \%$ confidence level; ${ }^{87} \mathrm{Rb}=1.42 \times 10^{-11}$ year ${ }^{-1}$. 


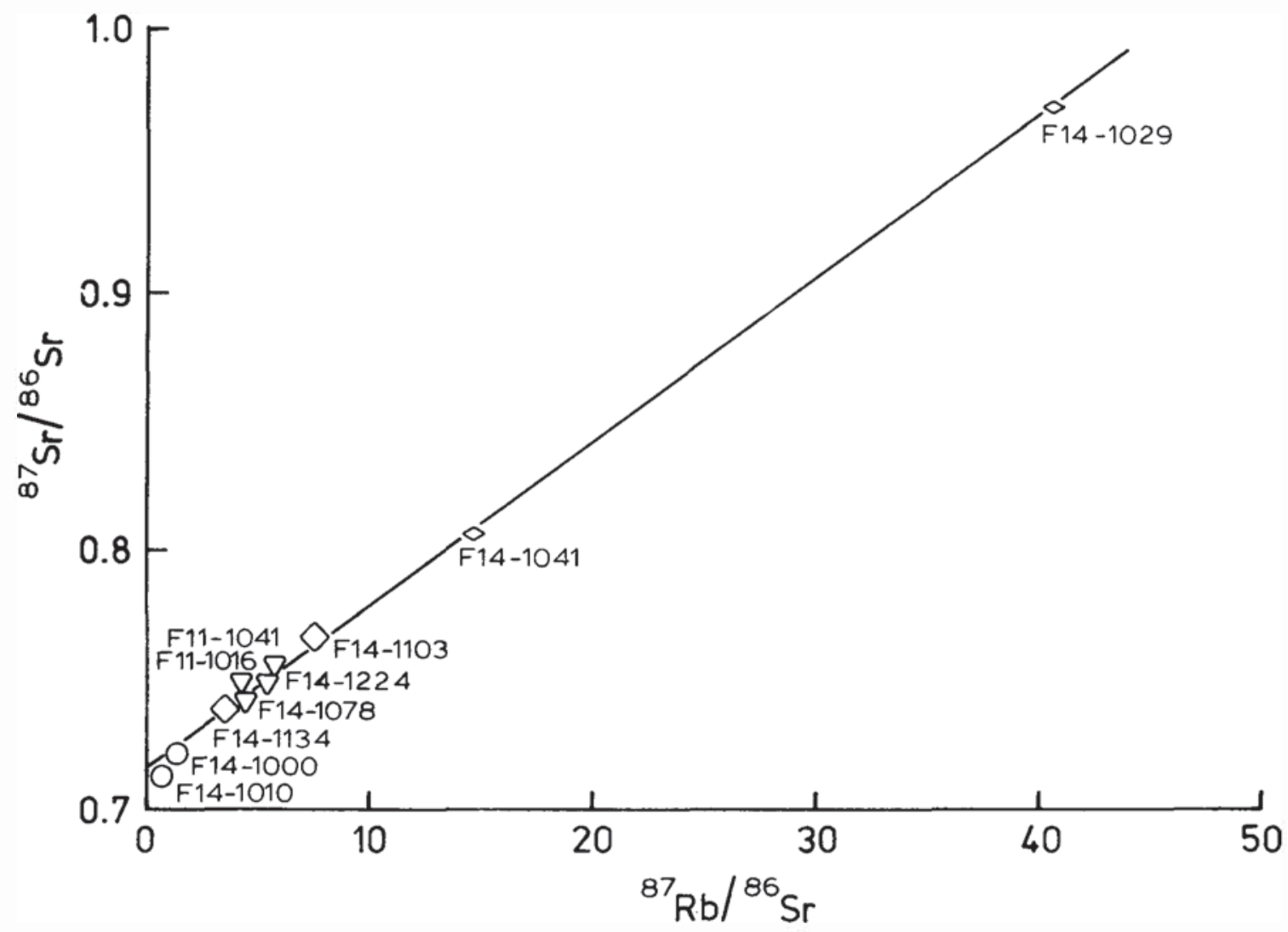

Fig. 6. Rb-Sr isochron calculated by method of York (1969) using data in Table 8. Errors at $95 \%$ confidence level. Decay constant for ${ }^{87} \mathrm{Rb}=1.42$ $x 10^{-11}$ /year. Initial ratio is $0.7156 \pm 0.0033$; age $=446 \pm 13 \mathrm{Ma}$ using all data.

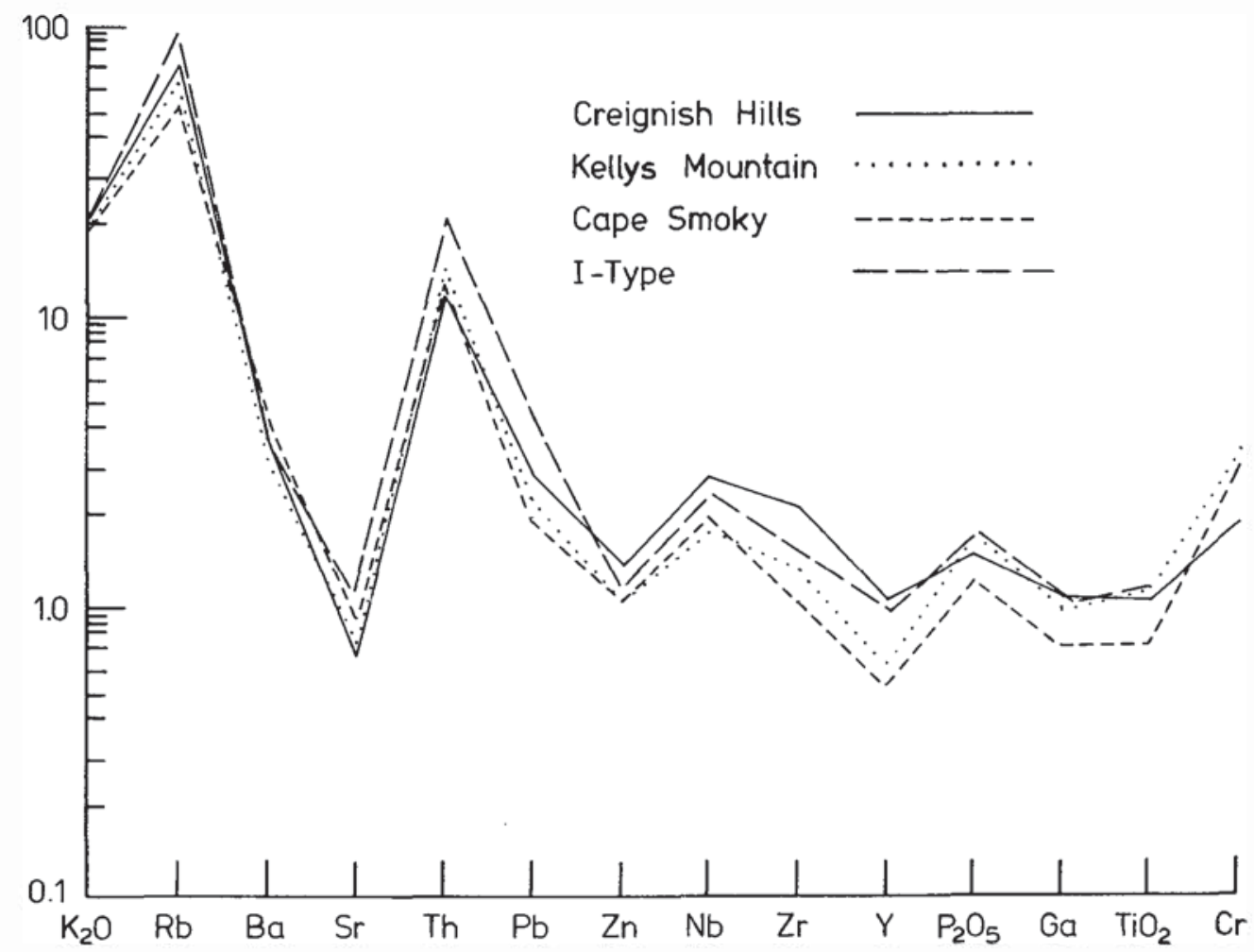

Fig. 7. Normalized oxide and element plot (spidergram) comparing average coarse-grained monzogranite (this study) to average Kellys Mountain leucogranite and Cape Smoky granite (calculated from data in Barr et al., 1982), and the average I-type granite from Whalen et al. (1987). Normalizing values from Pearce et al. (1984). 




Fig. 8. Temary plot of $\mathrm{FeO}$ (Total) $-\mathrm{Na}_{2} \mathrm{O}+\mathrm{K}_{2} \mathrm{O}-\mathrm{MgO}$. Tholeiitic-calcalkalic dividing line from Irvine and Baragar (1971).
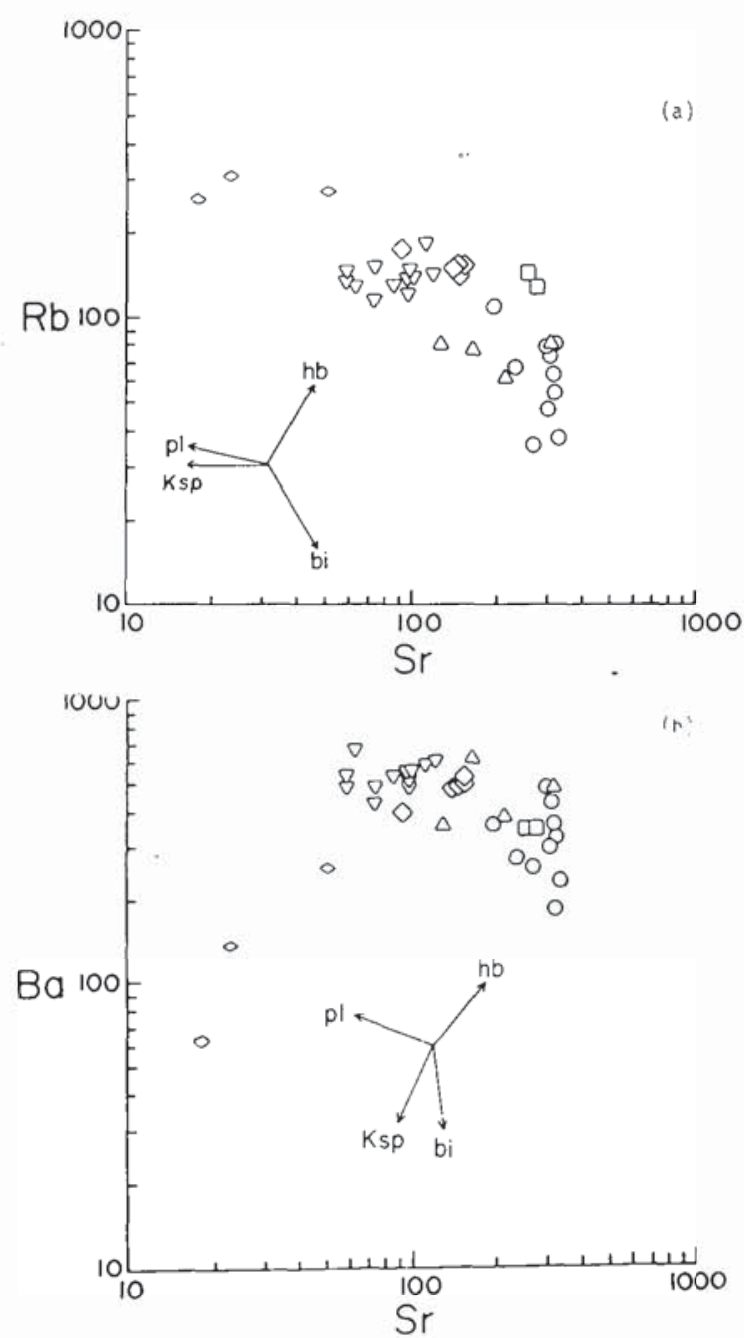

Fig. 9. Logarithmic plots of (a) Rb-Sr and (b) Ba-Sr. Approximate mineral fractionation trends after Tindle and Pearce (1981).

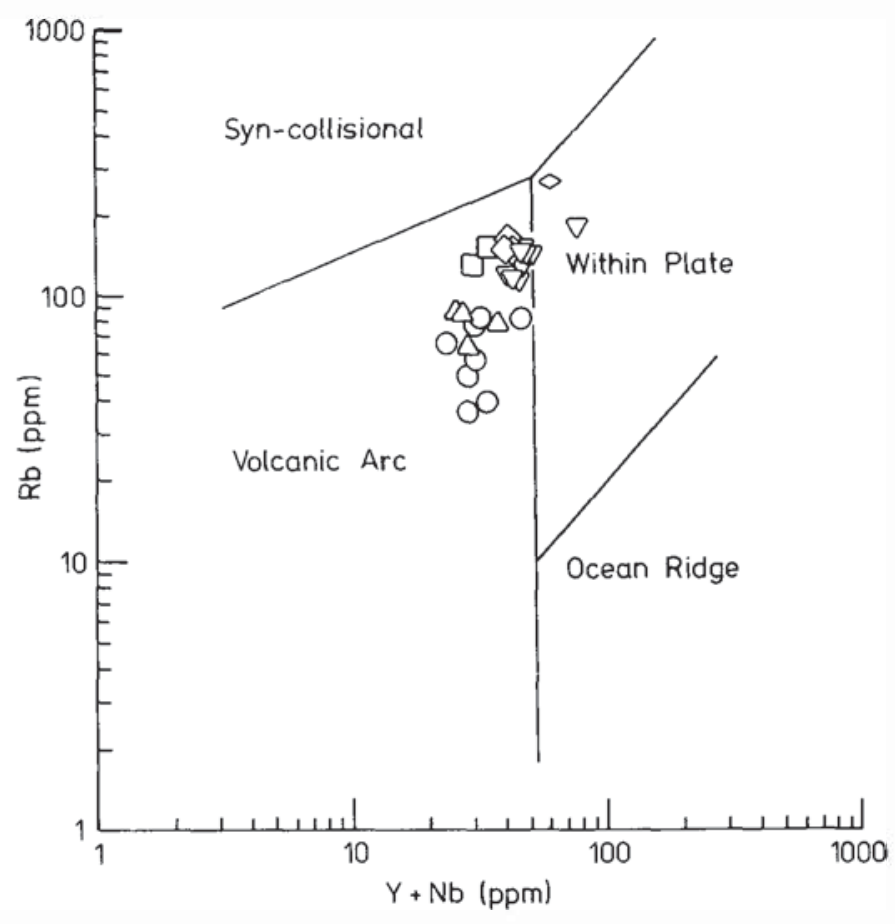

Fig. 10. Plot of Rb - Y + Nb. Fields from Pearce et al. (1984).

subduction continued to $495 \mathrm{Ma}$ in the Bras d'Or Terrane. These granites may have formed in response to localized thermal anomalies within the paleo-subduction zone.

\section{CONCLUSIONS}

This study has documented the field relationships and petrological characteristics of the units that comprise the Creignish Hills Pluton, enabling comparisons with other plutons of the Bras d'Or Terrane. The data suggest that the pluton is composite in terms of both lithology and age. It consists of a tonalite-diorite unit, postulated to have been emplaced at about $560 \mathrm{Ma}$, and units of granodiorite-tonalite, granodiorite-monzogranite, coarsegrained monzogranite and fine-grained monzogranite possibly emplaced at about $495 \mathrm{Ma}$. All the plutonic units appear to have formed as a result of subduction. However, in order to formulate more detailed interpretations of petrogenesis and regional tectonic implications, the age of the Creignish Hills Pluton has to be more reliably determined.

\section{ACKNOWLEDGEMENTS}

Most of the field work for this study was done by R.M. Campbell during May and June, 1979. The field work, thin section preparation, $\mathrm{Rb}$-Sr isotope studies and some geochemical analyses were funded by the Nova Scotia Department of Mines and Energy. Additional field studies and geochemical analyses were funded by an Operating Grant to S.M. Barr from the Natural Sciences and Engineering Research Council of Canada. We thank journal reviewers G. Pe-Piper and J. Hill for their constructive comments which resulted in significant improvements in the manuscript. 
ARMTTAGE, A.E. 1989. Geology and petrology of the crystalline rocks of the Whycocomagh area, Cape Breton Island, Nova Scotia. Unpublished B.Sc. thesis, Acadia University, Wolfville, Nova Scotia, 120 p.

BARR, S.M. and PRIDE, C.R. 1986. Petrogenesis of two contrasting Devonian granitic plutons, Cape Breton Island, Nova Scotia. Canadian Mineralogist, 24, pp. 137-146.

BARR, S.M. and RAESIDE, R.P. 1989. Tectono-stratigr aphic terranes in Cape Breton Island, Nova Scotia: Implications for the configuration of the northern Appalachian orogen. Geology, 17, pp. 822825.

BARR, S.M., DUNNING, G.R., RAESIDE, R.P., and JAMIESON, R.A. In press. Contrasting U-Pb ages from plutons in the $\mathrm{Bras}$ d' $\mathrm{Or}$ and Mira terranes of Cape Breton Island, Nova Scotia. Canadian Journal of Earth Sciences.

BARR, S.M., O'REILLY, G.A., and O'BEIRNE, A.M. 1982. Geology and geochemistry of selected granitoid plutons of Cape Breton Island. Nova Scotia Department of Mines and Energy, Paper 82 $1,176 \mathrm{p}$.

BLANCHARD, M-C. 1982. Geochemistry and petrogenesis of the Fissett Brook Formation, westem Cape Breton Island, Nova Scotia. Unpublished M.Sc. thesis, Dalhousie University, Halifax, Nova Scotia, 225 p.

CAMPBELL, J.E. and RAESIDE, R.P. 1990 . New ${ }^{40} \mathrm{Ar} /{ }^{39} \mathrm{Ar}$ ages of gneissic, greenschist and contact metamorphisms in the eastem Creignish Hills, Cape Breton Island, Nova Scotia. Abstract in Atlantic Geology, 26, pp. 167-168.

CAMPBELL, R.M. 1980. Creignish Hills pluton. In Mineral Resources Division Report of Activities, 1979. Nova Scotia Department of Mines and Energy, Report 80-1, pp. 111-115.

DUNNING, G.R., BARR, S.M., RAESIDE, R.P., and JAMIESON, R.A. 1989. U-Pb zircon, titanite, and monazite ages in the Bras d'Or and Aspy terranes of Cape Breton Island, Nova Scotia: Implications for magmatic and metamorphic history. Geological Society of America Bulletin, 102, pp. 322-330.

FERGUSON, S.A. and WEEKS, L.J. 1950. Mulgrave, Nova Scotia, Scale 1" = 1 mile; Geological Survey of Canada, Map 995A.

GIBSON, I.L. and JAGAM, P. 1980. Instrumental neutron activation analysis of rocks and minerals. In Neutron Activation Analyses in the Earth Sciences. Edited by G.K. Muecke. Mineralogical Association of Canada, Short Course Handbook, 5, pp. 109-131.

GORDON, G.E., RANDLE, K., GOLES, G.G., CORLISS, J.B., BEESON, M.H., and OXLEY, S.S. 1968. Instrumental activation analysis of standard rocks with high resolution gamma ray detectors. Geochimica et Cosmochimica Acta, 32, pp. 369-396.

HANSON, G.N. 1980. Rare earth elements in petrogenetic studies of igneous systems. Annual Reviews of Earth and Planetary Sciences, 8, pp. 371-406.

HASKIN, L.A., HASKIN, M.A., and FREY, F.A. 1968. Relative and absolute terrestrial abundances of the rare earths. In Origin and Distribution of the Elements. Edited by L.H. Ahrens. Pergamon, Oxford, pp. 889-912.

HUTCHISON, C.S. 1973. Laboratory handbook of petrographic techniques. Wiley, New York, 527 p.

IRVINE, T.N. and BARAGAR, W.R.A. 1971. A guide to the chemical classification of the common volcanic rocks. Canadian Journal of Earth Sciences, 8, pp. 523-548.
KELLEY, D.G. 1967. Baddeck and Whycocomagh map areas - with emphasis on Mississippian stratigraphy of Cape Breton Island. Geological Survey of Canada, Memoir 351, 65 p.

KELLEY, D.G. and MACKASEY, W.O. 1965. Basal Mississippian volcanic rocks in Cape Breton Island, Nova Scotia. Geological Survey of Canada, Paper 64-34, 10 p.

KEPPIE, J.D., MURPHY, J.B., NANCE, R.D., and DOSTAL, J. 1989. Terranes in Nova Scotia: Their characteristics and accretionary history. Nova Scotia Department of Mines and Energy, Report 89 3, pp. 117-122.

LEAKE, B.E. 1978. Nomenclature of amphiboles. Canadian Mineralogist, 16, pp. 501-520.

MILLIGAN, G.C. 1970. Geology of the George River Series, Cape Breton. Nova Scotia Department of Mines, Memoir 7, $111 \mathrm{p}$.

PEARCE, J.A., HARRIS, N.B.W., and TINDLE, A.G. 1984. Trace element discrimination diagrams for the tectonic interpretation of granitic rocks. Journal of Petrology, 25, pp. 956-983.

RAESIDE, R.P. and BARR, S.M. 1988. The Bras d'Or Zone: a suspect terrane in Cape Breton Island, Nova Scotia. Abstract in Program with Abstracts, Joint Annual Meeting, Geological Association of Canada-Mineralogical Association of Canada, 13, p. A102.

STRECKEISEN, A. 1976. To each plutonic rock its proper name. Earth Science Reviews, 12, pp. 1-23.

TINDLE, A.G. and PEARCE, J.A. 1981. Petrogenetic modelling of in situ fractional crystallization in the zoned Loch Doon Pluton, Scotland. Contributions to Mineralogy and Petrology, 78, pp. 196207.

WHALEN, J.B., CURRIE, K.L., and CHAPPELL, B.W. 1987. A-type granites: geochemical characteristics, discrimination and petrogenesis. Contributions to Mineralogy and Petrology, 95, pp. $407-$ 419.

YORK, D. 1969. Least squares fitting of a straight line with corrected errors. Earth and Planetary Sciences Letters, 5, pp. 320-324.

\section{APPENDIX A: Analytical Methods}

Major element analyses of most samples were done by atomic absorption spectrometry at Acadia University (analysts J. Cabilio and R.M. Campbell). Major elements in $\mathrm{CH}$ and JC samples were analyzed by X-ray fluorescence at the Regional XRF Facility, St. Mary's University, using fused disks. Analyses for As, F, Mo, S, Sn, U, Bi, and Li were done at CLIM Laboratories, Technical University of Nova Scotia, using methods described by Barr $e t$ al. (1982). A limited number of duplicate analyses by other methods suggest that these data should not be considered reliable in every case. Other trace elements were analyzed by X-ray fluorescence at the regional XRF Facility, St. Mary's University, using pressed powder pellets. Accuracy is considered to be generally within $\pm 10 \%$.

Rare-earth elements were analyzed by J.Loop at the University of Ottawa by instrumental neutron activation after the method described by Gordon et al. (1968), Gibson and Jagam (1980), and Barr and Pride (1986). Accuracy and precision are estimated to be $\pm 5 \%$. 